TRANSACTIONS OF THE

AMERICAN MATHEMATICAL SOCIETY

Volume 350, Number 8, August 1998, Pages 3297-3319

S 0002-9947(98)02058-3

\title{
QUANTIZED ENVELOPING ALGEBRAS FOR BORCHERDS SUPERALGEBRAS
}

\author{
GEORGIA BENKART, SEOK-JIN KANG, AND DUNCAN MELVILLE
}

\begin{abstract}
We construct quantum deformations of enveloping algebras of Borcherds superalgebras, their Verma modules, and their irreducible highest weight modules.
\end{abstract}

\section{INTRODUCTION}

Quantized enveloping algebras for Kac-Moody algebras were introduced independently by Drinfel'd [D] and Jimbo [Ji] in studying the quantum Yang-Baxter equation and two-dimensional solvable lattice models. The decade since then has seen a rich mathematical theory develop for these objects and their representations with connections to many areas of both mathematics and physics.

In 1988 Borcherds [B1] developed a generalization of Kac-Moody algebras to accommodate his study of monstrous moonshine and the vertex algebra representation of the monster simple group. These generalized Kac-Moody or Borcherds algebras, as they have become known, were originally described in terms of generators and relations which relax the defining conditions for Kac-Moody algebras. A major difference is that imaginary simple roots are allowed. In [B3] Borcherds gave a second characterization of them as Lie algebras with an almost positive symmetric contravariant bilinear form, and in [B5] he described a third characterization, which amounts to a recognition theorem. The most widely-studied examples of Borcherds algebras are the 'fake' monster [B2] and the monster [B4]. Recently Kang [Kn] has constructed quantum deformations for Borcherds algebras and their modules.

Lie superalgebras can be regarded as yet a third generalization of Kac-Moody algebras. Most attention so far has focused on the finite-dimensional simple Lie superalgebras, which were classified by Kac [Kc1]-[Kc2], and on their affine cousins, which have been shown to have important connections with number theory (see Kac-Wakimoto [KW1]-[KW2]). For many families of finite-dimensional Lie superalgebras such as the general linear, special linear, orthosymplectic, and Q-algebras, quantum deformations of their universal enveloping algebras have been constructed (see for example, Scheunert [S2], Floreanini et al. [FLV], Olshanski [O], Zou [Z], and Yamane $[\mathrm{Y}]$ (for affine superalgebras)). However, one of the difficulties that

Received by the editors October 1, 1996.

1991 Mathematics Subject Classification. Primary 17B37, 17B65, 17B67, 81R50.

The first author was supported in part by NSF Grant \#DMS-9300523.

The second author was supported in part by the Nondirected Research Fund, Korea Research Foundation, 1996.

The third author was supported in part by a Faculty Research Grant from St. Lawrence University. 
arises in constructing these deformations is determining what additional Serre relations are required in the presentation of the algebras. Thus far this problem has been addressed by treating specific families of finite-dimensional superalgebras, such as the ones cited above, in a case-by-case manner. For Kac-Moody Lie superalgebras defined by a symmetrizable Cartan matrix, Khoroshkin and Tolstoy [KT] have described quantized enveloping algebras and given an explicit expression for their universal $R$-matrix.

In this paper we weave these three strands together to discuss quantized enveloping algebras for Borcherds superalgebras whose Cartan matrix satisfies restrictions similar to those found in the paper by Kac [Kc2]. These conditions guarantee that the resulting algebras have a nondegenerate symmetric invariant bilinear form. Thus, included in the class of algebras we study are all the Kac-Moody superalgebras that appear in [Kc2] and the 'monstrous' and 'fake monstrous' Lie superalgebras, which are believed to have important connections with sporadic simple groups. Rather than assume we are working with a $\mathbf{Z}_{2}$-graded algebra, it is more convenient for us to suppose that the algebra is graded by an arbitrary abelian group (for example, the root lattice). Such algebras are often termed color algebras, but we will refer to them simply as superalgebras.

Section 1 contains all the necessary basic results on the structure and representations of Borcherds superalgebras, including the Weyl-Kac-Borcherds character formula, recently proven in this setting by Ray [Ra] and Miyamoto [M]. Additional background material can be found in the books of Chari and Pressley [CP], Jantzen [Ja], Kassel [Ks], and Lusztig [L2] for quantized enveloping algebras; in the survey [G] by Gebert for Borcherds algebras; and in the paper [S1] by Scheunert and the book [BMPZ] by Bahturin et al. for color algebras. In Section 2 we introduce super analogues of the $q$-binomial coefficients (see 2.6); construct a quantized enveloping algebra $U_{q}(\mathfrak{g})$ for the Borcherds superalgebra $\mathfrak{g}$ (Theorem 4.11 proves $U_{q}(\mathfrak{g})$ is actually a quantum deformation); and establish the standard properties of Verma modules for $U_{q}(\mathfrak{g})$. Using the A-form approach due originally to Lusztig [L1] for Kac-Moody algebras and to Kang [Kn] for generalized Kac-Moody algebras, we prove in Sections 3 and 4 that the quantum irreducible highest weight modules and quantum Verma modules are true deformations of the classical irreducible highest weight and Verma modules. Thus, for generic $q$, the dimensions of weight spaces are invariant under deformation, and the characters of the irreducible $U_{q}(\mathfrak{g})$-modules with dominant integral highest weights are given by the extensions of the Weyl-Kac-Borcherds formula due to Ray and Miyamoto.

Acknowledgment. We thank Jin Hong for his valuable comments on the original version of our manuscript.

\section{Borcherds Superalgebras}

1.1. Let $I$ be a countable (possibly infinite) index set. A matrix $A=\left(a_{i, j}\right)_{i, j \in I}$ with entries in the real numbers is a Borcherds-Cartan matrix if

(1.2). (i) $a_{i, i}=2$ or $a_{i, i} \leq 0$ for all $i \in I$,

(ii) $a_{i, j} \leq 0$ if $i \neq j$, and $a_{i, j} \in \mathbf{Z}$ if $a_{i, i}=2$,

(iii) $a_{i, j}=0$ if and only if $a_{j, i}=0$.

If there is a diagonal matrix $D=\operatorname{diag}\left(s_{i} \mid i \in I, s_{i}>0\right)$ such that $D A$ is symmetric, then $A$ is symmetrizable. A symmetrizable Borcherds-Cartan matrix $A$ is said to be integral if it further satisfies the following constraints: 
(1.3). (i) $a_{i, j} \in \mathbf{Z}$ for all $i, j \in I$,

(ii) $a_{i, i} \in 2 \mathbf{Z}$,

(iii) $s_{i} \in \mathbf{Z}_{>0}$.

1.4. A complex matrix $C=\left(\theta_{i, j}\right)_{i, j \in I}$ is a coloring matrix if $\theta_{i, j} \theta_{j, i}=1$ for all $i, j \in I$. Necessarily $\theta_{i, i}= \pm 1$, and we say $i$ is even when $\theta_{i, i}=1$, and $i$ is odd when $\theta_{i, i}=-1$. The Borcherds-Cartan matrix $A$ is restricted with respect to $C$ if $a_{i, i}=2$ and $\theta_{i, i}=-1$ imply that $a_{i, j} \in 2 \mathbf{Z}$ for all $j \in I$. For the matrices below, $A$ is restricted with respect to $C$ for any $c \neq 0$ :

$$
A=\left(\begin{array}{cc}
2 & -4 \\
-1 & -2
\end{array}\right), \quad C=\left(\begin{array}{cc}
-1 & c \\
c^{-1} & 1
\end{array}\right)
$$

1.5. Throughout this paper we assume $A$ is a symmetrizable integral BorcherdsCartan matrix which is restricted with respect to the coloring matrix $C$.

1.6. Suppose $P^{\vee}=\left(\bigoplus_{i \in I} \mathbf{Z} h_{i}\right) \oplus\left(\bigoplus_{i \in I} \mathbf{Z} d_{i}\right)$, and let $\mathfrak{h}=\mathbf{C} \otimes_{Z} P^{\vee}$ be the complex space with basis $\left\{h_{i}, d_{i} \mid i \in I\right\}$. For $i \in I$ we define $\alpha_{i}$ in the dual space $\mathfrak{h}^{*}$ of $\mathfrak{h}$ by setting $\alpha_{i}\left(h_{j}\right)=a_{j, i}$ and $\alpha_{i}\left(d_{j}\right)=\delta_{i, j}$.

1.7. The free abelian group $Q=\bigoplus_{i \in I} \mathbf{Z} \alpha_{i}$ generated by $\alpha_{i}$ 's $(i \in I)$ is the root lattice associated to $A$. Since $A$ is assumed to be symmetrizable, there exists a symmetric bilinear form (|) on $Q$ given by $\left(\alpha_{i} \mid \alpha_{j}\right)=s_{i} a_{i, j}=s_{j} a_{j, i}$. Let $Q^{+}=$ $\sum_{i \in I} \mathbf{Z}_{\geq 0} \alpha_{i}$ and $Q^{-}=-Q^{+}$. There is a partial ordering on $\mathfrak{h}^{*}$ in which $\lambda \geq \mu$ if and only if $\lambda-\mu \in Q^{+}$. The coloring matrix $C=\left(\theta_{i, j}\right)$ gives rise to a complex valued mapping $\theta: Q \times Q \longrightarrow \mathbf{C}^{\#}$ satisfying

(1.8). (i) $\theta\left(\alpha_{i}, \alpha_{j}\right)=\theta_{i, j}$,

(ii) $\theta(\alpha, \beta+\gamma)=\theta(\alpha, \beta) \theta(\alpha, \gamma)$,

(iii) $\theta(\alpha+\beta, \gamma)=\theta(\alpha, \gamma) \theta(\beta, \gamma)$,

for all $\alpha, \beta, \gamma \in Q$. These relations imply that $\theta(\alpha, \beta) \theta(\beta, \alpha)=1$ for all $\alpha, \beta$, and thus that $\theta(\alpha, \alpha)= \pm 1$. We say that $\alpha \in Q$ is even if $\theta(\alpha, \alpha)=1$ and odd if $\theta(\alpha, \alpha)=-1$. Then $\alpha_{i}$ is even (odd) if and only if $i$ is even (odd). Conditions (ii) and (iii) imply that $\theta(\alpha, 0)=1=\theta(0, \alpha)$ for all $\alpha \in Q$. In particular, 0 is even.

Definition 1.9. A complex vector space $L=\bigoplus_{\alpha \in Q} L_{\alpha}$ is a $\theta$-colored Lie superalgebra if there is a $\mathbf{C}$-bilinear product [, ] on $L$ such that $\left[L_{\alpha}, L_{\beta}\right] \subseteq L_{\alpha+\beta}$ and

$$
\begin{aligned}
& {[x, y]=-\theta(\alpha, \beta)[y, x]} \\
& {[x,[y, z]]=[[x, y], z]+\theta(\alpha, \beta)[y,[x, z]]}
\end{aligned}
$$

for all $x \in L_{\alpha}, y \in L_{\beta}$, and $z \in L$.

1.11. If $x \in L_{\alpha}$ in a $\theta$-colored Lie superalgebra, then $[x, x]=0$ when $\alpha$ is even and $[x,[x, x]]=0$ when $\alpha$ is odd. Suppose $L_{\bar{\ell}}=\bigoplus_{\alpha \in Q, \theta(\alpha, \alpha)=(-1)^{\ell}} L_{\alpha}$ for $\ell=0,1$. The relation $\theta(\alpha+\beta, \alpha+\beta)=\theta(\alpha, \alpha) \theta(\beta, \beta)$ shows that $L=L_{\overline{0}} \oplus L_{\overline{1}}$ is $\mathbb{Z}_{2}$-graded. Moreover, in the special case that $\theta(\alpha, \beta)=(-1)^{k \ell}$ whenever $\theta(\alpha, \alpha)=(-1)^{k}$ and $\theta(\beta, \beta)=(-1)^{\ell}$, the resulting algebra $L=L_{\overline{0}} \oplus L_{\overline{1}}$ is a Lie superalgebra. 
1.12. With respect to the Borcherds-Cartan matrix $A$, let $I^{r e}=\left\{i \in I \mid a_{i, i}=2\right\}$, $I^{i m}=\left\{i \in I \mid a_{i, i} \leq 0\right\}$. A collection of positive integers $\underline{m}=\left(m_{i} \mid i \in I\right)$ such that $m_{i}=1$ for all $i \in I^{r e}$ is called the charge of the matrix $A$. This brings us to the following definition, which is the superalgebra version of a Borcherds (or generalized Kac-Moody) Lie algebra.

Definition 1.13. Suppose $A$ is an integral Borcherds-Cartan matrix which is restricted with respect to the coloring matrix $C$. Then the Borcherds superalgebra $\mathfrak{g}(A, \underline{m}, C)=\sum_{\alpha \in Q} \mathfrak{g}_{\alpha}$ of charge $\underline{m}$ is the $\theta$-colored Lie superalgebra over $\mathbf{C}$ generated by the elements

$$
h_{i}, d_{i} \in \mathfrak{g}_{0} \quad(i \in I), \quad e_{i, k} \in \mathfrak{g}_{\alpha_{i}}, \quad f_{i, k} \in \mathfrak{g}_{-\alpha_{i}} \quad\left(i \in I, \quad k=1, \cdots, m_{i}\right)
$$

subject to the defining relations:

$$
\begin{aligned}
& {\left[h_{i}, h_{j}\right]=\left[h_{i}, d_{j}\right]=\left[d_{i}, d_{j}\right]=0,} \\
& {\left[h_{i}, e_{j, \ell}\right]=a_{i, j} e_{j, \ell}, \quad\left[h_{i}, f_{j, \ell}\right]=-a_{i, j} f_{j, \ell},} \\
& {\left[d_{i}, e_{j, \ell}\right]=\delta_{i, j} e_{j, \ell}, \quad\left[d_{i}, f_{j, \ell}\right]=-\delta_{i, j} f_{j, \ell},} \\
& {\left[e_{i, k}, f_{j, \ell}\right]=\delta_{i, j} \delta_{k, \ell} h_{i},} \\
& \left(\operatorname{ad} e_{i, k}\right)^{1-a_{i, j}}\left(e_{j, \ell}\right)=\left(\operatorname{ad} f_{i, k}\right)^{1-a_{i, j}}\left(f_{j, \ell}\right)=0 \text { if } a_{i, i}=2 \text { and } i \neq j, \\
& {\left[e_{i, k}, e_{j, \ell}\right]=\left[f_{i, k}, f_{j, \ell}\right]=0 \text { if } a_{i, j}=0,}
\end{aligned}
$$

for all $i, j \in I$, and $k=1, \cdots, m_{i}, \ell=1, \cdots, m_{j}$.

1.15. It follows from (1.14) that $\mathfrak{h}=\mathfrak{g}_{0}$ and $[h, x]=\alpha(h) x$ for all $x \in \mathfrak{g}_{\alpha}$. In particular, $\mathfrak{g}_{\alpha_{i}}=\mathbf{C} e_{i, 1} \oplus \cdots \oplus \mathbf{C} e_{i, m_{i}}$, and $\mathfrak{g}_{-\alpha_{i}}=\mathbf{C} f_{i, 1} \oplus \cdots \oplus \mathbf{C} f_{i, m_{i}}$. If $\mathfrak{g}_{\alpha} \neq(0)$ for $\alpha \neq 0$, then $\alpha \in Q$ is said to be a root and $\operatorname{dimg}_{\alpha}$ is its multiplicity. The relations in (1.14) imply that a root $\alpha$ must belong to $Q^{+}$or $Q^{-}$, and we denote by $\Phi, \Phi^{+}$, and $\Phi^{-}$the set of all roots, positive roots, and negative roots respectively. The subspaces $\mathfrak{g}^{ \pm}=\bigoplus_{\alpha \in \Phi^{ \pm}} \mathfrak{g}_{\alpha}$ are subalgebras of $\mathfrak{g}$, and they afford a triangular decomposition $\mathfrak{g}=\mathfrak{g}^{-} \oplus \mathfrak{h} \oplus \mathfrak{g}^{+}$of $\mathfrak{g}$. A root $\alpha$ is real if $(\alpha \mid \alpha)>0$, and imaginary if $(\alpha \mid \alpha) \leq 0$. The simple root $\alpha_{i}$ is real if $a_{i, i}=2$ (that is, if $i \in I^{r e}$ ), and imaginary if $a_{i, i} \leq 0\left(i \in I^{i m}\right)$. If $\alpha_{i}$ is an imaginary simple root, then its multiplicity is $m_{i}$. For each $i \in I^{r e}$, let $r_{i} \in \mathrm{GL}\left(\mathfrak{h}^{*}\right)$ be the simple reflection on $\mathfrak{h}^{*}$ defined by $r_{i}(\lambda)=\lambda-\lambda\left(h_{i}\right) \alpha_{i}$ for $\lambda \in \mathfrak{h}^{*}$. The subgroup $W$ of $\mathrm{GL}\left(\mathfrak{h}^{*}\right)$ generated by the $r_{i}$ 's $\left(i \in I^{\text {re }}\right)$ is the Weyl group of $\mathfrak{g}$.

1.16. The tensor algebra $T(L)$ of a $\theta$-colored Lie superalgebra $L$ is $Q$-graded. The universal enveloping algebra $U(L)$ of $L$ is obtained from $T(L)$ by factoring out by the ideal generated by the elements $[u, v]-u \otimes v+\theta(\alpha, \beta) v \otimes u$, where $u \in L_{\alpha}$ and $v \in L_{\beta}$. The universal enveloping algebra is a $Q$-graded associative algebra $U(L)=\bigoplus_{\alpha \in Q} U_{\alpha}$, containing $L$ with identity element $1 \in U_{0}$, and it has a Hopf superalgebra structure with comultiplication $\Delta$, counit $\epsilon$, and antipode $S$ such that

$$
\begin{array}{ll}
\Delta(1)=1 \otimes 1, & \Delta(x)=x \otimes 1+1 \otimes x, \\
\epsilon(1)=1, & \epsilon(x)=0, \\
S(1)=1, & S(x)=-x,
\end{array}
$$

for all $x \in L$. Note that in dealing with $\theta$-colored superalgebras, we have

$$
(a \otimes b)\left(a^{\prime} \otimes b^{\prime}\right)=\theta\left(\beta, \alpha^{\prime}\right) a a^{\prime} \otimes b b^{\prime}
$$

whenever $b$ lies in the $\beta$-graded subspace and $a^{\prime}$ in the $\alpha^{\prime}$-graded subspace. 
1.19. Suppose $\mathfrak{g}=\mathfrak{g}(A, \underline{m}, C)$ is the Borcherds superalgebra of charge $\underline{m}$ determined by $A$ and $C$. Then $U\left(\mathfrak{g}^{+}\right)$(resp. $U\left(\mathfrak{g}^{-}\right)$) is the C-subalgebra of $U(\mathfrak{g})$ with 1 generated by $e_{i, k}\left(\right.$ resp. $\left.f_{i, k}\right)$ for $i \in I, k=1, \cdots, m_{i}$ and $U(\mathfrak{g}) \cong U\left(\mathfrak{g}^{-}\right) \otimes U(\mathfrak{h}) \otimes$ $U\left(\mathfrak{g}^{+}\right)$. A $\mathfrak{g}$-module $V$ is said to be $\mathfrak{h}$-diagonalizable if it admits a weight space decomposition $V=\bigoplus_{\mu \in \mathfrak{h}^{*}} V_{\mu}$, where $V_{\mu}=\{v \in V \mid h \cdot v=\mu(h) v$ for all $h \in \mathfrak{h}\}$. If $\operatorname{dim}_{\mathbf{C}} V_{\mu}<\infty$ for all $\mu \in \mathfrak{h}^{*}$, then the character of $V$ is

$$
\operatorname{ch} V=\sum_{\mu \in \mathfrak{h}^{*}}\left(\operatorname{dim}_{\mathbf{C}} V_{\mu}\right) e^{\mu}
$$

where $e^{\mu}$ are the basis elements of the group algebra $\mathbf{C}\left[\mathfrak{h}^{*}\right]$ with the multiplication given by $e^{\mu} e^{\nu}=e^{\mu+\nu}$ for $\mu, \nu \in \mathfrak{h}^{*}$. An $\mathfrak{h}$-diagonalizable $\mathfrak{g}$-module $V$ is a highest weight module with highest weight $\lambda \in \mathfrak{h}^{*}$ if there is a nonzero vector $v_{\lambda} \in V$ (a highest weight vector) such that (i) $e_{i, k} \cdot v_{\lambda}=0$ for all $i \in I, k=1, \cdots, m_{i}$, (ii) $h \cdot v_{\lambda}=\lambda(h) v_{\lambda}$ for all $h \in \mathfrak{h}$, (iii) $V=U(\mathfrak{g}) \cdot v_{\lambda}$. For a highest weight module $V$ with highest weight $\lambda$, we have (i) $V=U\left(\mathfrak{g}^{-}\right) \cdot v_{\lambda}$, (ii) $V=\bigoplus_{\mu \leq \lambda} V_{\mu}$ where $V_{\lambda}=\mathbf{C} v_{\lambda}$, and (iii) $\operatorname{dim}_{\mathbf{C}} V_{\mu}<\infty$ for all $\mu$.

1.20. Let $\lambda \in \mathfrak{h}^{*}$ and consider the left ideal $J(\lambda)$ of $U(\mathfrak{g})$ generated by the elements $e_{i, k}\left(i \in I, k=1, \cdots, m_{i}\right)$ and $h-\lambda(h) 1(h \in \mathfrak{h})$. Then $M(\lambda)=U(\mathfrak{g}) / J(\lambda)$ is a left $U(\mathfrak{g})$-module which is a highest weight module with highest weight $\lambda$ and highest weight vector $v_{\lambda}=1+J(\lambda)$. The $U(\mathfrak{g})$-module $M(\lambda)$ is the Verma module with highest weight $\lambda$. The standard facts about Verma modules can be proved in this context:

Proposition 1.21. (a) Every highest weight module over $U(\mathfrak{g})$ with highest weight $\lambda \in \mathfrak{h}^{*}$ is a homomorphic image of $M(\lambda)$.

(b) As a $U\left(\mathfrak{g}^{-}\right)$-module, $M(\lambda)$ is free of rank one generated by the highest weight vector $v_{\lambda}=1+J(\lambda)$.

(c) $M(\lambda)$ contains a unique maximal submodule $N(\lambda)$ and has a unique irreducible quotient $V(\lambda)=M(\lambda) / N(\lambda)$. Every irreducible highest weight module of weight $\lambda$ is isomorphic to $V(\lambda)$.

1.22. Let

$$
P=\left\{\lambda \in \mathfrak{h}^{*} \mid \lambda\left(h_{i}\right) \in \mathbf{Z}, \lambda\left(d_{i}\right) \in \mathbf{Z} \text { for all } i \in I\right\} .
$$

$P$ is called the weight lattice of $\mathfrak{g}$ with respect to $\mathfrak{h}$. An element $\lambda \in P$ is said to be a dominant integral weight if

$$
\begin{aligned}
& \lambda\left(h_{i}\right) \in \mathbf{Z}_{\geq 0} \text { for all } i \in I, \\
& \lambda\left(h_{i}\right) \in 2 \mathbf{Z}_{\geq 0} \text { for all } i \in I^{r e} \cap I^{\text {odd }},
\end{aligned}
$$

where $I^{\text {odd }}$ denotes the set of $i \in I$ such that $\theta\left(\alpha_{i}, \alpha_{i}\right)=\theta_{i, i}=-1$. Let $P^{+}$denote the set of dominant integral weights.

1.25. Suppose $\rho \in \mathfrak{h}^{*}$ satisfies $\rho\left(h_{i}\right)=\frac{1}{2} a_{i, i}$ for all $i \in I$, and let $R$ be the set of all imaginary simple roots counted with multiplicities. When $A$ is restricted with respect to $C$, Ray $[\mathrm{Ra}]$ and Miyamoto $[\mathrm{M}]$ have established the following generalization of the Weyl-Kac-Borcherds formula for the character of the irreducible highest weight module $V(\lambda)$ with highest weight $\lambda \in P^{+}$for the Borcherds superalgebra $\mathfrak{g}(A, \underline{m}, C)$ : 
Theorem 1.26 ([Ra, Theorem 2.3], [M, Theorem 9.1]). For $\lambda \in P^{+}$, let

$$
S_{\lambda}=e^{\lambda+\rho} \sum_{\beta}(-1)^{|\beta|} e^{-\beta},
$$

where $\beta$ runs over all the elements of the weight lattice $P$ of the form

$$
\beta=\alpha_{i_{1}}+\cdots+\alpha_{i_{r}}+p_{j_{1}} \beta_{j_{1}}+\cdots+p_{j_{s}} \beta_{j_{s}} \quad(r=s=0 \quad \text { if } \beta=0)
$$

such that $\alpha_{i_{k}}$ (resp. $\beta_{j_{l}}$ ) are distinct even (resp. odd) imaginary simple roots of $R$ satisfying $\left(\alpha_{i_{k}} \mid \alpha_{i_{l}}\right)=\left(\beta_{j_{k}} \mid \beta_{j_{l}}\right)=0$ if $k \neq l,\left(\alpha_{i_{k}} \mid \beta_{j_{l}}\right)=0$ for all $k, l,\left(\beta_{j_{k}} \mid \beta_{j_{k}}\right)=0$ if $p_{j_{k}} \geq 2$, and $\left(\lambda \mid \alpha_{i_{k}}\right)=\left(\lambda \mid \beta_{j_{l}}\right)=0$ for all $k, l$. For such $a \beta$, we denote $|\beta|=$ $r+\sum_{k=1}^{s} p_{j_{k}}$.

Then we have

$$
\operatorname{ch} V(\lambda)=\frac{e^{-\rho} \sum_{w \in W}(-1)^{l(w)} w\left(S_{\lambda}\right)}{\prod_{\alpha \in \Phi^{-}}\left(1-\theta(\alpha, \alpha) e^{\alpha}\right)^{\theta(\alpha, \alpha) \operatorname{dim} \mathfrak{g}_{\alpha}} .}
$$

Corollary 1.27. Assume that $V$ is a highest weight module over $U(\mathfrak{g})$ with highest weight $\lambda \in P^{+}$and highest weight vector $v_{\lambda}$ such that

(i) if $\lambda\left(h_{i}\right)=0$, then $f_{i, k} \cdot v_{\lambda}=0$ for $k=1, \cdots, m_{i}$,

(ii) if $a_{i, i}=2$, then $f_{i, k}^{\lambda\left(h_{i}\right)+1} \cdot v_{\lambda}=0$.

Then $V$ is isomorphic to the irreducible highest weight module $V(\lambda)$.

\section{A Quantum Deformation of $\mathfrak{g}(A, \underline{m}, C)$}

2.1. In this section we construct a deformation $U_{q}(\mathfrak{g})$ of the Borcherds superalgebra $\mathfrak{g}=\mathfrak{g}(A, \underline{m}, C)$, and in subsequent sections we show that highest weight modules for $\mathfrak{g}$ deform to highest weight modules for $U_{q}(\mathfrak{g})$.

2.2. Let us begin by defining the $q$-commutator for a Lie color algebra to be

$$
[x, y]_{q}=x y-q^{(\alpha \mid \beta)} \theta(\alpha, \beta) y x
$$

for $x$ of degree $\alpha$ and $y$ of degree $\beta$, and by denoting the corresponding adjoint mapping by $\operatorname{ad}_{q}(x)(y)=[x, y]_{q}$. Since $[y, x]_{q}=y x-q^{(\beta \mid \alpha)} \theta(\beta, \alpha) x y=$ $-q^{(\beta \mid \alpha)} \theta(\beta, \alpha)\left(x y-q^{-(\alpha \mid \beta)} \theta(\alpha, \beta) y x\right)$, it follows that the relation

$$
[y, x]_{q}=-q^{(\beta \mid \alpha)} \theta(\beta, \alpha)[x, y]_{q^{-1}}
$$

holds. Applying (2.3) and induction, we have in the case $a_{i, i}=2$ that

$$
\left(\operatorname{ad}_{q}\left(e_{i, k}\right)\right)^{N}\left(e_{j, \ell}\right)=\sum_{n=0}^{N}(-1)^{n} \theta_{i, j}^{n} \theta_{i, i}^{n(n-1) / 2} q_{i}^{n a_{i, j}+N n-n}\left\{\begin{array}{l}
N \\
n
\end{array}\right\}_{q_{i}} e_{i, k}^{N-n} e_{j, \ell} e_{i, k}^{n}
$$

where $q_{i}=q^{s_{i}}$ and $\theta_{i, j}=\theta\left(\alpha_{i}, \alpha_{j}\right)$. The binomial coefficients are defined by

$$
\{n\}_{q_{i}}=\frac{\theta_{i, i}^{n} q_{i}^{n}-q_{i}^{-n}}{\theta_{i, i} q_{i}-q_{i}^{-1}}, \quad\{n\}_{q_{i}} !=\prod_{m=1}^{n}\{m\}_{q_{i}}, \quad\left\{\begin{array}{c}
N \\
n
\end{array}\right\}_{q_{i}}=\frac{\{N\}_{q_{i}} !}{\{n\}_{q_{i}} !\{N-n\}_{q_{i}} !},
$$

where $\{0\}_{q_{i}} !=1$.

Definition 2.7. Suppose $\mathfrak{g}=\mathfrak{g}(A, \underline{m}, C)$ is the Borcherds superalgebra of charge $\underline{m}$ determined by the symmetrizable integral Borcherds-Cartan matrix $A$ which is restricted with respect to the coloring matrix $C$. Assume $q$ is an indeterminate. Then the quantum algebra $U_{q}(\mathfrak{g})$ associated to $\mathfrak{g}$ is the associative algebra over $\mathbf{C}(q)$ with 1 generated by the elements $q^{h}\left(h \in P^{\vee}\right), e_{i, k}, f_{i, k}\left(i \in I, k=1, \cdots, m_{i}\right)$ with defining relations 
(2.8). (i) $q^{0}=1, q^{h} q^{h^{\prime}}=q^{h+h^{\prime}} \quad$ for $h, h^{\prime} \in P^{\vee}$;

(ii) $q^{h} e_{i, k} q^{-h}=q^{\alpha_{i}(h)} e_{i, k}, \quad q^{h} f_{i, k} q^{-h}=q^{-\alpha_{i}(h)} f_{i, k}$

for $h \in P^{\vee}, i \in I, k=1, \cdots, m_{i}$;

(iii) $e_{i, k} f_{j, \ell}-\theta_{j, i} f_{j, \ell} e_{i, k}=\delta_{i, j} \delta_{k, \ell} \frac{K_{i}-K_{i}^{-1}}{q_{i}-q_{i}^{-1}}$,

where $q_{i}=q^{s_{i}}$ and $K_{i}=q^{s_{i} h_{i}}$

for $i, j \in I, k=1, \cdots, m_{i}, \ell=1, \cdots, m_{j}$;

(iv) $\left(\operatorname{ad}_{q}\left(e_{i, k}\right)\right)^{1-a_{i, j}}\left(e_{j, \ell}\right)$

$=\sum_{n=0}^{1-a_{i, j}}(-1)^{n} \theta_{i, j}^{n} \theta_{i, i}^{n(n-1) / 2}\left\{\begin{array}{c}1-a_{i, j} \\ n\end{array}\right\}_{q_{i}} e_{i, k}^{1-a_{i, j}-n} e_{j, \ell} e_{i, k}^{n}=0$

if $a_{i, i}=2$ and $i \neq j$;

(v) $\left(\operatorname{ad}_{q}\left(f_{i, k}\right)\right)^{1-a_{i, j}}\left(f_{j, \ell}\right)$

$=\sum_{n=0}^{1-a_{i, j}}(-1)^{n} \theta_{i, j}^{n} \theta_{i, i}^{n(n-1) / 2}\left\{\begin{array}{c}1-a_{i, j} \\ n\end{array}\right\}_{q_{i}} f_{i, k}^{1-a_{i, j}-n} f_{j, \ell} f_{i, k}^{n}=0$

if $a_{i, i}=2$ and $i \neq j$;

(vi) $e_{i, k} e_{j, \ell}-\theta_{i, j} e_{j, \ell} e_{i, k}=0 \quad$ if $a_{i, j}=0$

(vii) $f_{i, k} f_{j, \ell}-\theta_{i, j} f_{j, \ell} f_{i, k}=0 \quad$ if $a_{i, j}=0$,

where $\theta_{i, j}=\theta\left(\alpha_{i}, \alpha_{j}\right)$ is the $(i, j)$ entry of the coloring matrix $C$.

Proposition 2.9. The algebra $U_{q}(\mathfrak{g})$ has a Hopf superalgebra structure with comultiplication $\Delta$, counit $\varepsilon$, and antipode $S$ defined by

$$
\begin{aligned}
& \Delta\left(q^{h}\right)=q^{h} \otimes q^{h} \quad \text { for } h \in P^{\vee}, \\
& \Delta\left(e_{i, k}\right)=e_{i, k} \otimes K_{i}^{-1}+1 \otimes e_{i, k}, \\
& \Delta\left(f_{i, k}\right)=f_{i, k} \otimes 1+K_{i} \otimes f_{i, k} \quad \text { for } i \in I, k=1, \cdots, m_{i}, \\
& \quad \varepsilon\left(q^{h}\right)=1 \text { for } h \in P^{\vee}, \\
& \quad \varepsilon\left(e_{i, k}\right)=\varepsilon\left(f_{i, k}\right)=0 \quad \text { for } i \in I, k=1, \cdots, m_{i},
\end{aligned}
$$

$$
S\left(q^{h}\right)=q^{-h} \text { for } h \in P^{\vee},
$$$$
S\left(e_{i, k}\right)=-e_{i, k} K_{i}, \quad S\left(f_{i, k}\right)=-K_{i}^{-1} f_{i, k} \quad \text { for } i \in I, k=1, \cdots, m_{i} .
$$

Proof. The assertions can be verified by defining these maps on the generators $q^{h}$ $\left(h \in P^{\vee}\right), e_{i, k}, f_{i, k}\left(i \in I, k=1, \cdots, m_{i}\right)$ as in (2.10)-(2.12), extending them to the free associative algebra with 1 over $\mathbf{C}(q)$ by requiring that $\Delta$ and $\varepsilon$ be algebra morphisms and $S$ be a $\theta$-colored antimorphism (that is, $S(a b)=\theta(\alpha, \beta) S(b) S(a)$ whenever $a \in U_{q}(\mathfrak{g})_{\alpha}$ and $\left.b \in U_{q}(\mathfrak{g})_{\beta}\right)$, and then showing that they preserve the relations in (2.8). All of this is straightforward, save perhaps the calculations involving relations (iv) and (v). We illustrate the arguments in these cases.

Using the fact that the antipode is a $\theta$-colored antimorphism and equation (2.4) above, we have for any $x$ of degree $\alpha$ and $y$ of degree $\beta$ that

$$
S\left(\left(\operatorname{ad}_{q}(x)\right)^{N}(y)\right)=(-1)^{N} q^{(\alpha \mid \beta) N+(\alpha \mid \alpha) N(N-1) / 2}\left(\operatorname{ad}_{q^{-1}}(S(x))\right)^{N}(S(y)) .
$$

Let us apply this now when $x=e_{i, k}$ and $a_{i, i}=2, y=e_{j, \ell}$, and $N=1-a_{i, j}$, and use the fact that $S\left(e_{i, k}\right)=-e_{i, k} K_{i}$, which has the same degree as $e_{i, k}$. We will 
also make use of the fact that $q^{\left(\alpha_{i} \mid \alpha_{j}\right)}=q^{s_{i} a_{i, j}}=q_{i}^{a_{i, j}}$. Then

$$
\begin{aligned}
S\left(\left(\operatorname{ad}_{q}\left(e_{i, k}\right)\right)^{N}\right. & \left.\left(e_{j, \ell}\right)\right)=(-1)^{N} q_{i}^{(1-N) N+2 N(N-1) / 2}\left(\operatorname{ad}_{q^{-1}}\left(S\left(e_{i, k}\right)\right)\right)^{N}\left(S\left(e_{j, \ell}\right)\right) \\
& =(-1)^{N}\left(\operatorname{ad}_{q^{-1}}\left(S\left(e_{i, k}\right)\right)\right)^{N}\left(S\left(e_{j, \ell}\right)\right) \\
& =(-1)^{N} \sum_{n=0}^{N}(-1)^{n} \theta_{i, j}^{n} \theta_{i, i}^{n(n-1) / 2}\left\{\begin{array}{c}
N \\
n
\end{array}\right\}_{q_{i}^{-1}} S\left(e_{i, k}\right)^{N-n} S\left(e_{j, \ell}\right) S\left(e_{i, k}\right)^{n} .
\end{aligned}
$$

To unravel this, note that

$$
\begin{aligned}
S\left(e_{i, k}\right)^{N-n} S\left(e_{j, \ell}\right) S\left(e_{i, k}\right)^{n} & =(-1)^{N+1}\left(e_{i, k} K_{i}\right)^{N-n} e_{j, \ell} K_{j}\left(e_{i, k} K_{i}\right)^{n} \\
& =(-1)^{N+1} q^{\left(\alpha_{i} \mid \alpha_{j}\right) N+\left(\alpha_{i} \mid \alpha_{i}\right) N(N-1) / 2} e_{i, k}^{N-n} e_{j, \ell} e_{i, k}^{n} K_{i}^{N} K_{j} \\
& =(-1)^{N+1} e_{i, k}^{N-n} e_{j, \ell} e_{i, k}^{n} K_{i}^{N} K_{j} .
\end{aligned}
$$

Also observe that

$$
\left\{\begin{array}{l}
N \\
n
\end{array}\right\}_{q_{i}^{-1}}=\theta_{i, i}^{n(N-n)}\left\{\begin{array}{l}
N \\
n
\end{array}\right\}_{q_{i}} .
$$

We would like to argue that $\theta_{i, i}^{n(N-n)}=1$ when $N=1-a_{i, j}$. Now if $\theta_{i, i}=1$, this is clear. If instead $\theta_{i, i}=-1$, then $a_{i, j} \in 2 \mathbf{Z}$ for all $j$, so that $N=1-a_{i, j}$ is odd. If $n$ is even, $\theta_{i, i}^{n(N-n)}=1$. If $n$ is odd, then $N-n$ is even, and again the expression is 1 . Thus,

$$
\left\{\begin{array}{c}
N \\
n
\end{array}\right\}_{q_{i}^{-1}}=\left\{\begin{array}{l}
N \\
n
\end{array}\right\}_{q_{i}}
$$

under the hypothesis $N=1-a_{i, j}$. Putting this in, we have for $N=1-a_{i, j}$,

$$
\begin{aligned}
& S\left(\left(\operatorname{ad}_{q}\left(e_{i, k}\right)\right)^{N}\left(e_{j, \ell}\right)\right) \\
& =(-1)^{N} \sum_{n=0}^{N}(-1)^{n} \theta_{i, j}^{n} \theta_{i, i}^{n(n-1) / 2}\left\{\begin{array}{c}
N \\
n
\end{array}\right\}_{q_{i}^{-1}} S\left(e_{i, k}\right)^{N-n} S\left(e_{j, \ell}\right) S\left(e_{i, k}\right)^{n} \\
& =-\left(\sum_{n=0}^{N}(-1)^{n} \theta_{i, j}^{n} \theta_{i, i}^{n(n-1) / 2}\left\{\begin{array}{l}
N \\
n
\end{array}\right\}_{q_{i}} e_{i, k}^{N-n} e_{j, \ell} e_{i, k}^{n}\right) K_{i}^{N} K_{j}=0,
\end{aligned}
$$

which shows that the antipode preserves the Serre relation.

Now to prove the comultiplication preserves the Serre relation, first note that by induction,

$$
\begin{aligned}
\Delta\left(\left(\operatorname{ad}_{q}\left(e_{i, k}\right)\right)^{N}\right. & \left.\left(e_{j, l}\right)\right)=\left(\operatorname{ad}_{q}\left(e_{i, k}\right)\right)^{N}\left(e_{j, l}\right) \otimes K_{i}^{-N} K_{j}^{-1} \\
& +\sum_{n=0}^{N-1} \tau_{n}^{(N)} q_{i}^{n(N-n)}\left\{\begin{array}{l}
N \\
n
\end{array}\right\}_{q_{i}} e_{i, k}^{N-n} \otimes K_{i}^{-N+n}\left(\operatorname{ad}_{q}\left(e_{i, k}\right)\right)^{n}\left(e_{j, l}\right) \\
& +1 \otimes\left(\operatorname{ad}_{q}\left(e_{i, k}\right)\right)^{N}\left(e_{j, l}\right),
\end{aligned}
$$

where $\tau_{n}^{(N)}=\prod_{t=n}^{N-1}\left(1-\theta_{i, i}^{t} q_{i}^{2\left(t+a_{i, j}\right)}\right)$. If $N=1-a_{i, j}$, then $1-\theta_{i, i}^{N-1} q_{i}^{2\left(N-1+a_{i, j}\right)}=$ $1-\theta_{i, i}^{-a_{i, j}}$. Recall that $a_{i, j} \in 2 \mathbf{Z}$ if $\theta_{i, i}=-1$. Hence $1-\theta_{i, i}^{-a_{i, j}}=0$, which implies 
$\tau_{n}^{(N)}=0$ for all $n=0,1, \cdots, N-1$, so that all the middle terms drop out. Therefore, the comultiplication $\Delta$ preserves the Serre relation.

2.14. The $\mathbf{C}(q)$-subalgebra $U_{q}^{0}$ of $U_{q}(\mathfrak{g})$ generated by the elements $q^{h}\left(h \in P^{\vee}\right)$ is the group algebra $\mathbf{C}(q)\left[P^{\vee}\right]$. Assume $U_{q}^{+}$(resp. $U_{q}^{-}$) is the $\mathbf{C}(q)$-subalgebra of $U_{q}(\mathfrak{g})$ with 1 generated by the elements $e_{i, k}\left(\right.$ resp. $f_{i, k}$ ) for $i \in I, k=1, \cdots, m_{i}$.

2.15. We define a $\mathbf{C}(q)$-linear transformation $T$ on $U_{q}(\mathfrak{g})$ by first specifying that

$$
\begin{aligned}
& T\left(q^{h}\right)=q^{-h} \text { for } h \in P^{\vee}, \\
& T\left(e_{i, k}\right)=\theta_{i, i} f_{i, k}, \quad T\left(f_{i, k}\right)=e_{i, k} \quad \text { for } i \in I, k=1, \cdots, m_{i},
\end{aligned}
$$

and then extending $T$ to the free associative algebra with 1 on those generators by requiring that $T$ is an algebra morphism, i.e. $T(a b)=T(a) T(b)$. It is not difficult to check that the relations in (2.8) are preserved by $T$, and hence that $T$ induces an algebra morphism on $U_{q}(\mathfrak{g})$. Moreover, $T$ satisfies the properties

$$
\begin{aligned}
& T^{4}=\mathrm{id}, \\
& \Delta \circ T=\sigma \circ(T \otimes T) \circ \Delta, \\
& \varepsilon \circ T=\varepsilon, \\
& T \circ S=d \circ S \circ T,
\end{aligned}
$$

where $\sigma$ is the twist map $\sigma(a \otimes b)=b \otimes a$, and $d$ is the degree map $d(a)=q^{(\alpha \mid \alpha)} a$ if $a \in U_{q}(\mathfrak{g})_{\alpha}$. The second property says that $T$ is a coalgebra morphism. It is apparent that the following holds:

Lemma 2.18. The mapping $T$ gives an algebra isomorphism between $U_{q}^{-}$and $U_{q}^{+}$.

2.19. We will use the next result along with ideas of Rosso [Ro] to argue that $U_{q}(\mathfrak{g})$ admits a triangular decomposition $U_{q}(\mathfrak{g}) \cong U_{q}^{-} \otimes U_{q}^{0} \otimes U_{q}^{+}$.

Lemma 2.20. Let $U_{q}^{\geq 0}$ (resp. $U_{q}^{\leq 0}$ ) denote the $\boldsymbol{C}(q)$-subalgebra of $U_{q}(\mathfrak{g})$ generated by the elements $q^{h}\left(h \in P^{\vee}\right.$ ) and the elements $e_{i, k}$ (resp. $f_{i, k}$ ) for $i \in I, k=$ $1, \ldots, m_{i}$. Then

$$
\begin{aligned}
& U_{q}^{\geq 0} \cong U_{q}^{0} \otimes U_{q}^{+}, \\
& U_{q}^{\leq 0} \cong U_{q}^{-} \otimes U_{q}^{0} .
\end{aligned}
$$

Proof. We present an argument for the second one and obtain the first from that by applying $T$. Let $\left\{f_{\zeta} \mid \zeta \in \Omega\right\}$ denote a basis for $U_{q}^{-}$consisting of monomials $f_{\zeta}$ in the elements $f_{i, k}$, where $\Omega$ is just a set indexing the basis elements. The defining relations imply that the elements $f_{\zeta} q^{h}\left(\zeta \in \Omega, h \in P^{\vee}\right)$ span $U_{q}^{\leq 0}$. There is a surjective $\mathbf{C}(q)$-linear map $U_{q}^{-} \otimes U_{q}^{0} \longrightarrow U_{q}^{\leq 0}$ given by $f_{\zeta} \otimes q^{h} \longrightarrow f_{\zeta} q^{h}$. To show injectivity of this map, it suffices to show that the elements $f_{\zeta} q^{h}\left(\zeta \in \Omega, h \in P^{\vee}\right)$ are linearly independent over $\mathbf{C}(q)$. Then

Suppose $\sum_{\zeta \in \Omega, h \in P^{\vee}} c_{\zeta, h} f_{\zeta} q^{h}=0$ is a dependence relation with $c_{\zeta, h} \in \mathbf{C}(q)$.

$$
\sum_{\beta \in Q^{-}}\left(\sum_{\operatorname{deg} f_{\zeta}=\beta, h \in P^{\vee}} c_{\zeta, h} f_{\zeta} q^{h}\right)=0,
$$


which implies that

$$
\sum_{\operatorname{deg} f_{\zeta}=\beta, h \in P^{\vee}} c_{\zeta, h} f_{\zeta} q^{h}=0
$$

for each $\beta$. Suppose $\beta=-\sum_{i \in I} k_{i} \alpha_{i}\left(k_{i} \in \mathbf{Z}_{\geq 0}\right)$, and let $h_{\beta}=\sum_{i \in I} k_{i} s_{i} h_{i}$. Since $f_{\zeta}$ is a monomial in the $f_{i, k}$ 's, we have

$$
\Delta\left(f_{\zeta}\right)=f_{\zeta} \otimes 1+(\text { intermediate terms })+q^{h_{\beta}} \otimes f_{\zeta} .
$$

Therefore, applying $\Delta$ to $(2.22)$ gives

$$
\begin{aligned}
0 & =\sum_{\operatorname{deg} f_{\zeta}=\beta, h \in P^{\vee}} c_{\zeta, h}\left(f_{\zeta} \otimes 1+(\text { intermediate terms })+q^{h_{\beta}} \otimes f_{\zeta}\right)\left(q^{h} \otimes q^{h}\right) \\
& =\sum_{\operatorname{deg} f_{\zeta}=\beta, h \in P^{\vee}} c_{\zeta, h}\left(f_{\zeta} q^{h} \otimes q^{h}+(\text { intermediate terms })+q^{h_{\beta}+h} \otimes f_{\zeta} q^{h}\right) .
\end{aligned}
$$

The terms of degree $(0, \beta)$ must sum to zero, so

$$
\sum_{\operatorname{deg} f_{\zeta}=\beta, h \in P^{\vee}} c_{\zeta, h} q^{h_{\beta}+h} \otimes f_{\zeta} q^{h}=0 .
$$

But since the elements $q^{h_{\beta}+h}$ are linearly independent, this implies that $\sum_{\operatorname{deg} f_{\zeta}=\beta} c_{\zeta, h} f_{\zeta} q^{h}=0$. Consequently $\sum_{\operatorname{deg} f_{\zeta}=\beta} c_{\zeta, h} f_{\zeta}=0$, and thus $c_{\zeta, h}=0$ for all $\zeta \in \Omega, h \in P^{\vee}$.

Theorem 2.23. There is a $C(q)$-linear isomorphism $U_{q}(\mathfrak{g}) \cong U_{q}^{-} \otimes U_{q}^{0} \otimes U_{q}^{+}$.

Proof. As in the previous lemma, it suffices to show that the elements $f_{\zeta} q^{h} e_{\eta}$ $\left(\zeta, \eta \in \Omega, h \in P^{\vee}\right)$ are linearly independent over $\mathbf{C}(q)$. From the dependence relation $\sum_{\zeta, \eta \in \Omega, h \in P^{\vee}} c_{\zeta, h, \eta} f_{\zeta} q^{h} e_{\eta}=0$ we obtain

$$
\sum_{h \in P^{\vee}, \operatorname{deg} f_{\zeta}+\operatorname{deg} e_{\eta}=\gamma} c_{\zeta, h, \eta} f_{\zeta} q^{h} e_{\eta}=0
$$

for each $\gamma \in Q$. Now when $\operatorname{deg} f_{\zeta}=\alpha=-\sum_{i \in I} k_{i} \alpha_{i}$, we write $h_{\zeta}=h_{\alpha}=$ $\sum_{i \in I} k_{i} s_{i} h_{i}$; and similarly when $\operatorname{deg} e_{\eta}=\beta=\sum_{i \in I} m_{i} \alpha_{i}$, we set $h_{\eta}=h_{\beta}=$ $\sum_{i \in I} m_{i} s_{i} h_{i}$. Then

$$
\begin{aligned}
& \Delta\left(e_{\eta}\right)=e_{\eta} \otimes q^{-h_{\eta}}+(\text { intermediate terms })+1 \otimes e_{\eta}, \\
& \Delta\left(f_{\zeta}\right)=f_{\zeta} \otimes 1+(\text { intermediate terms })+q^{h_{\zeta}} \otimes f_{\zeta} .
\end{aligned}
$$

Therefore,

$$
\begin{aligned}
& 0=\Delta\left(\sum_{h \in P^{\vee}, \operatorname{deg} f_{\zeta}+\operatorname{deg} e_{\eta}=\gamma} c_{\zeta, h, \eta} f_{\zeta} q^{h} e_{\eta}\right) \\
&=\sum_{h \in P^{\vee}, \operatorname{deg} f_{\zeta}+\operatorname{deg} e_{\eta}=\gamma} c_{\zeta, h, \eta}\left(f_{\zeta} \otimes 1+(\text { intermediate terms })+q^{h_{\zeta}} \otimes f_{\zeta}\right) \\
& \quad \times\left(q^{h} \otimes q^{h}\right)\left(e_{\eta} \otimes q^{-h_{\eta}}+(\text { intermediate terms })+1 \otimes e_{\eta}\right) .
\end{aligned}
$$

Consider the total ordering $\leq$ on $Q$, first by height and then, within a given height, by a lexicographical ordering with respect to the $\alpha_{i}$ 's. Suppose $\widetilde{\Omega}$ is the set of all $\zeta \in \Omega$ such that $\operatorname{deg} f_{\zeta}$ is minimal among the terms in (2.24), and let $\widetilde{\Omega}^{\prime}$ be the ones 
among the $\eta \in \Omega$ in (2.24) with $\operatorname{deg} e_{\eta}$ maximal. Since $\operatorname{deg} f_{\zeta}+\operatorname{deg} e_{\eta}=\gamma$, it is clear that $\zeta \in \widetilde{\Omega}$ if and only if $\eta \in \widetilde{\Omega}^{\prime}$. As a result, the terms in $(2.24)$ of degree $(\alpha, \beta)$ with $\alpha \in Q^{-}$minimal and $\beta \in Q^{+}$maximal must sum to zero. Hence,

$$
\sum_{h \in P^{\vee}, \operatorname{deg} f_{\zeta}=\alpha, \operatorname{deg} e_{\eta}=\beta} q^{h_{\alpha}+h} e_{\eta} \otimes f_{\zeta} q^{h-h_{\beta}}=0 .
$$

Since the vectors $f_{\zeta} q^{h-h_{\beta}}\left(\zeta \in \widetilde{\Omega}, h \in P^{\vee}\right)$ are linearly independent by Lemma 2.20, $\sum_{h \in P^{\vee}, \operatorname{deg} e_{\eta}=\beta} c_{\zeta, h, \eta} q^{h_{\alpha}+h} e_{\eta}=0$. Again using Lemma 2.20, we have $c_{\zeta, h, \eta}=0$ for all $\zeta \in \widetilde{\Omega}, h \in P^{\vee}, \eta \in \widetilde{\Omega}^{\prime}$. Repeating this argument with the remaining terms, we conclude that $c_{\zeta, h, \eta}=0$ for all $\zeta, \eta \in \Omega, h \in P^{\vee}$.

2.25. A $U_{q}(\mathfrak{g})$-module $V^{q}$ is said to be diagonalizable if it admits a weight space decomposition $V^{q}=\bigoplus_{\mu \in P} V_{\mu}^{q}$, where $V_{\mu}^{q}=\left\{v \in V^{q} \mid q^{h} \cdot v=q^{\mu(h)} v\right.$ for all $\left.h \in P^{\vee}\right\}$. If $V^{q}$ is diagonalizable, then so is every submodule $X^{q}$. Indeed, this can be argued inductively by supposing $x=x_{1}+\cdots+x_{p} \in X^{q}$, with $x_{i} \in V_{\mu_{i}}^{q}\left(\mu_{i} \neq \mu_{j}\right.$ if $i \neq j$ ), and every element with fewer summands has its weight components in $X^{q}$. Then $\sum_{i=2}^{p}\left(q^{\mu_{1}(h)}-q^{\mu_{i}(h)}\right) x_{i}=q^{\mu_{1}(h)} x-q^{h} x \in X^{q}$, and, by minimality, $\left(q^{\mu_{1}(h)}-q^{\mu_{i}(h)}\right) x_{i} \in X^{q}$ for all $i \neq 1$. Since the weights $\mu_{i}$ are distinct, and $q$ is not a root of unity, this forces $x_{i} \in X^{q}$ for all $i$. Thus, $X^{q}=\bigoplus_{\mu \in P} X_{\mu}^{q}$, where $X_{\mu}^{q}=X^{q} \cap V_{\mu}^{q}$.

2.26. If $\operatorname{dim}_{\mathbf{C}(q)} V_{\mu}^{q}<\infty$ for all $\mu \in P$, then the character of $V^{q}$ is defined to be

$$
\operatorname{ch} V^{q}=\sum_{\mu \in P}\left(\operatorname{dim}_{\mathbf{C}(q)} V_{\mu}^{q}\right) e^{\mu},
$$

where $e^{\mu}$ are the basis elements of the group algebra $\mathbf{C}(q)[P]$ with the multiplication given by $e^{\mu} e^{\nu}=e^{\mu+\nu}$ for $\mu, \nu \in P$. A diagonalizable $U_{q}(\mathfrak{g})$-module $V^{q}$ is a highest weight module with highest weight $\lambda \in P$ if there is a nonzero vector $v_{\lambda} \in V^{q}$ (a highest weight vector) such that (i) $e_{i, k} \cdot v_{\lambda}=0$ for all $i \in I, k=1, \cdots, m_{i}$, (ii) $q^{h} \cdot v_{\lambda}=q^{\lambda(h)} v_{\lambda}$ for all $h \in P^{\vee}$, (iii) $V^{q}=U_{q}(\mathfrak{g}) \cdot v_{\lambda}$. When $V^{q}$ is a highest weight $U_{q}(\mathfrak{g})$-module with highest weight $\lambda \in P$, then (i) $V^{q}=U_{q}^{-} \cdot v_{\lambda}$, (ii) $V^{q}=\bigoplus_{\mu \leq \lambda} V_{\mu}^{q}$, $V_{\lambda}^{q}=\mathbf{C}(q) v_{\lambda}$, and (iii) $\operatorname{dim}_{\mathbf{C}(q)} V_{\mu}^{q}<\infty$ for all $\mu \leq \lambda$.

2.27. Assume $\lambda \in P$ and consider the left ideal $I_{q}(\lambda)$ of $U_{q}(\mathfrak{g})$ generated by the elements $e_{i, k}\left(i \in I, k=1, \cdots, m_{i}\right)$ and $q^{h}-q^{\lambda(h)} 1\left(h \in P^{\vee}\right)$. Then $M^{q}(\lambda) \stackrel{\text { def }}{=}$ $U_{q}(\mathfrak{g}) / I_{q}(\lambda)$ is a highest weight $U_{q}(\mathfrak{g})$-module under left multiplication with highest weight $\lambda$ and highest weight vector $v_{\lambda}=1+I_{q}(\lambda)$. It is the Verma module with highest weight $\lambda$ for $U_{q}(\mathfrak{g})$.

Proposition 2.28 (cf. [Kc3, Proposition 9.2]). (a) For every $\lambda \in P$, every highest weight module over $U_{q}(\mathfrak{g})$ with highest weight $\lambda$ is a homomorphic image of $M^{q}(\lambda)$.

(b) As a $U_{q}^{-}$-module, $M^{q}(\lambda)$ is free of rank one generated by the highest weight vector $v_{\lambda}=1+I_{q}(\lambda)$.

(c) $M^{q}(\lambda)$ contains a unique maximal submodule $N^{q}(\lambda)$.

Proof. (a) If $V^{q}$ is a highest weight $U_{q}(\mathfrak{g})$-module with highest weight $\lambda$ and highest weight vector $w_{\lambda}$, then the map $M^{q}(\lambda) \rightarrow V^{q}$ given by $u \cdot\left(1+I_{q}(\lambda)\right) \mapsto u \cdot w_{\lambda}$ defines a surjective $U_{q}(\mathfrak{g})$-module homomorphism.

(b) Since every element $u$ of $U_{q}(\mathfrak{g})$ can be written as a sum of elements of the form $u^{-} u^{0} u^{+}$, where $u^{ \pm} \in U_{q}^{ \pm}$and $u^{0} \in U_{q}^{0}$, every element of $M^{q}(\lambda)$ has the form 
$u^{-} \cdot\left(1+I_{q}(\lambda)\right)$ for some $u^{-} \in U_{q}\left(\mathfrak{g}^{-}\right)$. If $u^{-} \cdot\left(1+I_{q}(\lambda)\right)=0$, then $u^{-} \in I_{q}(\lambda)$, which is generated by $e_{i, k}\left(i \in I, k=1, \cdots, m_{i}\right)$ and $q^{h}-q^{\lambda(h)} 1\left(h \in P^{\vee}\right)$. Hence $u^{-}$must be zero, and our assertion follows.

(c) Note that for any proper submodule $M^{\prime}$ of $M^{q}(\lambda), M^{\prime} \subseteq \bigoplus_{\mu \in P, \mu \neq \lambda} V_{\mu}^{q}$. Thus the sum of proper submodules is again a proper submodule of $M^{q}(\lambda)$. Let $N^{q}(\lambda)$ be the sum of all the proper submodules of $M^{q}(\lambda)$. Then $N^{q}(\lambda)$ is the unique maximal submodule of $M^{q}(\lambda)$.

2.29. For $\lambda \in P$, the unique irreducible quotient $V^{q}(\lambda) \stackrel{\text { def }}{=} M^{q}(\lambda) / N^{q}(\lambda)$ is the irreducible highest weight module over $U_{q}(\mathfrak{g})$ with highest weight $\lambda$.

\section{A-FORMS}

3.1. Let $A=\left(a_{i, j}\right)_{i, j \in I}$ be a symmetrizable integral Borcherds-Cartan matrix with charge $\underline{m}=\left(m_{i} \mid i \in I\right)$ which is restricted with respect to the coloring matrix $C=\left(\theta_{i, j}\right)_{i, j \in I}$, and let $U_{q}(\mathfrak{g})$ be the quantum algebra associated with the Borcherds superalgebra $\mathfrak{g}=\mathfrak{g}(A, \underline{m}, C)$. Recall that $q_{i}=q^{s_{i}}$ and $K_{i}=q^{s_{i} h_{i}}$ for $i \in I$. Assume that $D_{i}=q^{s_{i} d_{i}}(i \in I)$. Then for $i, j \in I$ and $k=1, \cdots, m_{j}$ we have

$$
\begin{array}{ll}
K_{i} e_{j, k} K_{i}^{-1}=q_{i}^{a_{i, j}} e_{j, k}, & K_{i} f_{j, k} K_{i}^{-1}=q_{i}^{-a_{i, j}} f_{j, k}, \\
D_{i} e_{j, k} D_{i}^{-1}=q_{i}^{\delta_{i, j}} e_{j, k}, & D_{i} f_{j, k} D_{i}^{-1}=q_{i}^{-\delta_{i, j}} f_{j, k} .
\end{array}
$$

For $i \in I, c \in \mathbf{Z}, n \in \mathbf{Z}_{\geq 0}$, we adopt the following conventions used in [L1]:

$$
\begin{aligned}
& {\left[\begin{array}{c}
K_{i} ; c \\
n
\end{array}\right]=\prod_{r=1}^{n} \frac{K_{i} q_{i}^{c-r+1}-K_{i}^{-1} q_{i}^{-(c-r+1)}}{q_{i}^{r}-q_{i}^{-r}},} \\
& {\left[\begin{array}{c}
D_{i} ; c \\
n
\end{array}\right]=\prod_{r=1}^{n} \frac{D_{i} q_{i}^{c-r+1}-D_{i}^{-1} q_{i}^{-(c-r+1)}}{q_{i}^{r}-q_{i}^{-r}} .}
\end{aligned}
$$

3.5 The "ordinary" binomial coefficients in $q_{i}$ are defined by

$$
\begin{aligned}
{[n]_{q_{i}}=\frac{q_{i}^{n}-q_{i}^{-n}}{q_{i}-q_{i}^{-1}}, \quad[n]_{q_{i}} ! } & =\prod_{m=1}^{n}[m]_{q_{i}}, \quad\left[\begin{array}{c}
N \\
n
\end{array}\right]_{q_{i}}=\frac{[N]_{q_{i}} !}{[n]_{q_{i}} ![N-n]_{q_{i}} !}, \\
{\left[\begin{array}{c}
-N \\
n
\end{array}\right]_{q_{i}} } & =(-1)^{n}\left[\begin{array}{c}
N+n-1 \\
n
\end{array}\right]_{q_{i}},
\end{aligned}
$$

where $N>0, n \geq 0$, and $[0]_{q_{i}} !=1$. Then since

$$
\begin{aligned}
& \frac{K_{i} q_{i}^{c-r+1}-K_{i}^{-1} q_{i}^{-(c-r+1)}}{q_{i}^{r}-q_{i}^{-r}} \\
& =\frac{K_{i} q_{i}^{c-r+1}-K_{i}^{-1} q_{i}^{c-r+1}+K_{i}^{-1} q_{i}^{c-r+1}-K_{i}^{-1} q_{i}^{-(c-r+1)}}{q_{i}^{r}-q_{i}^{-r}} \\
& =\frac{q_{i}^{c-r+1}\left(K_{i}-K_{i}^{-1}\right)}{q_{i}^{r}-q_{i}^{-r}}+K_{i}^{-1} \frac{q_{i}^{c-r+1}-q_{i}^{-(c-r+1)}}{q_{i}^{r}-q_{i}^{-r}} \\
& =\frac{q_{i}^{c-r+1}\left(q_{i}-q_{i}^{-1}\right)}{q_{i}^{r}-q_{i}^{-r}} \frac{K_{i}-K_{i}^{-1}}{q_{i}-q_{i}^{-1}}+K_{i}^{-1} \frac{q_{i}^{c-r+1}-q_{i}^{-(c-r+1)}}{q_{i}-q_{i}^{-1}} \frac{q_{i}-q_{i}^{-1}}{q_{i}^{r}-q_{i}^{-r}} \\
& =\frac{1}{[r]_{q_{i}}}\left(q_{i}^{c-r+1}\left[\begin{array}{c}
K_{i} \\
1
\end{array}\right]+[c-r+1]_{q_{i}} K_{i}^{-1}\right),
\end{aligned}
$$


we have

$$
\left[\begin{array}{c}
K_{i} ; c \\
n
\end{array}\right]=\prod_{r=1}^{n} \frac{1}{[r]_{q_{i}}}\left(q_{i}^{c-r+1}\left[\begin{array}{c}
K_{i} ; 0 \\
1
\end{array}\right]+[c-r+1]_{q_{i}} K_{i}^{-1}\right)
$$

for all $c \in \mathbf{Z}$, and an analogous relation holds with $D_{i}$ in place of $K_{i}$.

3.9. Let $\mathbf{A}=\mathbf{C}\left[q, q^{-1}, 1 /[n]_{q_{i}}, i \in I, n>0\right]$ and define the $\mathbf{A}$-form $U_{\mathbf{A}}$ of the quantum algebra $U_{q}(\mathfrak{g})$ to be the A-subalgebra of $U_{q}(\mathfrak{g})$ with 1 generated by the elements $e_{i, k}, f_{i, k}\left(i \in I, k=1, \cdots, m_{i}\right), q^{h}\left(h \in P^{\vee}\right),\left[\begin{array}{c}K_{i} ; 0 \\ 1\end{array}\right]=\frac{K_{i}-K_{i}^{-1}}{q_{i}-q_{i}^{-1}}$, $\left[\begin{array}{cc}D_{i} ; & 0 \\ 1\end{array}\right]=\frac{D_{i}-D_{i}^{-1}}{q_{i}-q_{i}^{-1}}(i \in I)$. We denote by $U_{\mathbf{A}}^{+}$(resp. $\left.U_{\mathbf{A}}^{-}\right)$the A-subalgebra of $U_{q}(\mathfrak{g})$ with 1 generated by $e_{i, k},\left(\operatorname{resp} . f_{i, k}\right)$ for $i \in I, k=1, \cdots, m_{i}$, and by $U_{\mathbf{A}}^{0}$ the A-subalgebra of $U_{q}(\mathfrak{g})$ with 1 generated by $q^{h}\left(h \in P^{\vee}\right),\left[\begin{array}{c}K_{i} ; \\ 1\end{array}\right],\left[\begin{array}{cc}D_{i} ; & 0 \\ 1\end{array}\right](i \in I)$. In particular, from (3.8) we see that $\left[\begin{array}{c}K_{i} ; c \\ n\end{array}\right]$ and $\left[\begin{array}{c}D_{i} ; c \\ n\end{array}\right]$ belong to $U_{\mathbf{A}}^{0}$ for all $c \in \mathbf{Z}, n \in \mathbf{Z}_{>0}$. Moreover, the following commutation relations hold in $U_{\mathbf{A}}$ :

Lemma 3.10. For $i, j \in I, k=1, \cdots, m_{i}, \ell=1, \cdots, m_{j}, c \in \boldsymbol{Z}$, and $n \in \boldsymbol{Z}_{>0}$, we have

$$
\begin{gathered}
e_{j, \ell}\left[\begin{array}{c}
K_{i} ; c \\
n
\end{array}\right]=\left[\begin{array}{c}
K_{i} ; c-a_{i, j} \\
n
\end{array}\right] e_{j, \ell}, \\
{\left[\begin{array}{c}
K_{i} ; c \\
n
\end{array}\right] f_{j, \ell}=f_{j, \ell}\left[\begin{array}{c}
K_{i} ; c-a_{i, j} \\
n
\end{array}\right],} \\
e_{j, \ell}\left[\begin{array}{c}
D_{i} ; c \\
n
\end{array}\right]=\left[\begin{array}{c}
D_{i} ; c-\delta_{i, j} \\
n
\end{array}\right] e_{j, \ell}, \\
{\left[\begin{array}{c}
D_{i} ; c \\
n
\end{array}\right] f_{j, \ell}=f_{j, \ell}\left[\begin{array}{c}
D_{i} ; c-\delta_{i, j} \\
n
\end{array}\right],} \\
e_{i, k} f_{j, \ell}=\theta_{i, j} f_{j, \ell} e_{i, k} \text { if } i \neq j \text { or } k \neq l, \\
e_{i, k} f_{i, k}^{n}=\theta_{i, i}^{n} f_{i, k}^{n} e_{i, k}+f_{i, k}^{n-1} \sum_{r=0}^{n-1} \theta_{i, i}^{n-1-r}\left[\begin{array}{c}
K_{i} ;-r a_{i, i} \\
1
\end{array}\right] .
\end{gathered}
$$

Proof. Equations (3.11)-(3.15) follow directly from the defining relations of $U_{q}(\mathfrak{g})$ and (3.2)-(3.4), while (3.16) can be proved by induction.

3.17. As an immediate consequence of Lemma 3.10 we have the triangular decomposition of the algebra $U_{\mathbf{A}}$ :

$$
U_{\mathbf{A}} \cong U_{\mathbf{A}}^{-} \otimes U_{\mathbf{A}}^{0} \otimes U_{\mathbf{A}}^{+}
$$

In particular, every element $u$ of $U_{\mathbf{A}}$ can be written as a sum of monomials of the form $u^{-} u^{0} u^{+}$, where $u^{0} \in U_{\mathbf{A}}^{0}, u^{ \pm} \in U_{\mathbf{A}}^{ \pm}$.

Corollary 3.19. Let $V^{q}(\lambda)$ be the irreducible highest weight $U_{q}(\mathfrak{g})$-module with highest weight $\lambda \in P^{+}$and highest weight vector $v_{\lambda}$.

(a) If $\lambda\left(h_{i}\right)=0$, then $f_{i, k} \cdot v_{\lambda}=0$ for $k=1, \cdots, m_{i}$.

(b) If $a_{i, i}=2$, then $f_{i, k}^{\lambda\left(h_{i}\right)+1} \cdot v_{\lambda}=0$. 
Proof. (a) If $k \neq \ell$, then $e_{i, \ell} f_{i, k} \cdot v_{\lambda}=\theta_{i, i} f_{i, k} e_{i, \ell} \cdot v_{\lambda}=0$. If $k=\ell$, then since $\lambda\left(h_{i}\right)=0$ we have

$$
\begin{aligned}
e_{i, k} f_{i, k} \cdot v_{\lambda} & =\theta_{i, i} f_{i, k} e_{i, k} \cdot v_{\lambda}+\frac{K_{i}-K_{i}^{-1}}{q_{i}-q_{i}^{-1}} v_{\lambda} \\
& =\frac{q_{i}^{\lambda\left(h_{i}\right)}-q_{i}^{-\lambda\left(h_{i}\right)}}{q_{i}-q_{i}^{-1}} v_{\lambda}=0 .
\end{aligned}
$$

For $j \neq i, e_{j, \ell} f_{i, k} \cdot v_{\lambda}=\theta_{j, i} f_{i, k} e_{j, \ell} \cdot v_{\lambda}=0$ for $\ell=1, \cdots, m_{j}$. Hence $f_{i, k} \cdot v_{\lambda}$ is a primitive vector in $V^{q}(\lambda)$. Since $V^{q}(\lambda)$ is irreducible, it must be that $f_{i, k} \cdot v_{\lambda}=0$, for otherwise $f_{i, k} \cdot v_{\lambda}$ would generate a proper submodule of $V^{q}(\lambda)$ with highest weight $\lambda-\alpha_{i} \neq \lambda$, which is a contradiction.

(b) If $a_{i, i}=2$, then $\lambda\left(h_{i}\right) \in \mathbf{Z}_{\geq 0}$ for $i \in I^{\text {even }}$ and $\lambda\left(h_{i}\right) \in 2 \mathbf{Z}_{\geq 0}$ for $i \in I^{\text {odd }}$, since $\lambda \in P^{+}$.

Suppose $i \in I^{\text {even }}$. Then by (3.16) we have

$$
\begin{aligned}
& e_{i, k} f_{i, k}^{\lambda\left(h_{i}\right)+1} \cdot v_{\lambda}=f_{i, k}^{\lambda\left(h_{i}\right)+1} e_{i, k} \cdot v_{\lambda}+f_{i, k}^{\lambda\left(h_{i}\right)} \sum_{r=0}^{\lambda\left(h_{i}\right)}\left[\begin{array}{c}
K_{i} ;-2 r \\
1
\end{array}\right] \cdot v_{\lambda} \\
& =\sum_{r=0}^{\lambda\left(h_{i}\right)} f_{i, k}^{\lambda\left(h_{i}\right)} \frac{q^{\lambda\left(h_{i}\right)-2 r}-q^{-\lambda\left(h_{i}\right)+2 r}}{q_{i}-q_{i}^{-1}} v_{\lambda} \\
& =\left(q_{i}-q_{i}^{-1}\right)^{-1}\left(\left(q_{i}^{\lambda\left(h_{i}\right)}-q_{i}^{-\lambda\left(h_{i}\right)}\right)+\left(q_{i}^{\lambda\left(h_{i}\right)-2}-q_{i}^{-\lambda\left(h_{i}\right)+2}\right)\right. \\
& \left.\quad+\cdots+\left(q_{i}^{-\lambda\left(h_{i}\right)+2}-q_{i}^{\lambda\left(h_{i}\right)-2}\right)+\left(q_{i}^{-\lambda\left(h_{i}\right)}-q_{i}^{\lambda\left(h_{i}\right)}\right)\right) f_{i}^{\lambda\left(h_{i}\right)} \cdot v_{\lambda}=0 .
\end{aligned}
$$

If $i \in I^{\text {odd }}$, then $\lambda\left(h_{i}\right) \in 2 \mathbf{Z}_{\geq 0}$ and $\theta_{i, i}=-1$. Hence relation (3.16) yields

$$
\begin{aligned}
& e_{i, k} f_{i, k}^{\lambda\left(h_{i}\right)+1} \cdot v_{\lambda}=(-1)^{\lambda\left(h_{i}\right)+1} f_{i, k}^{\lambda\left(h_{i}\right)+1} e_{i, k} \cdot v_{\lambda} \\
& \quad+f_{i, k}^{\lambda\left(h_{i}\right)} \sum_{r=0}^{\lambda\left(h_{i}\right)}(-1)^{\lambda\left(h_{i}\right)-r}\left[\begin{array}{c}
K_{i} ;-2 r \\
1
\end{array}\right] \cdot v_{\lambda} \\
& =\sum_{r=0}^{\lambda\left(h_{i}\right)} f_{i, k}^{\lambda\left(h_{i}\right)}(-1)^{\lambda\left(h_{i}\right)-r} \frac{q^{\lambda\left(h_{i}\right)-2 r}-q^{-\lambda\left(h_{i}\right)+2 r}}{q_{i}-q_{i}^{-1}} v_{\lambda} \\
& =\left(q_{i}-q_{i}^{-1}\right)^{-1}\left(\left(q_{i}^{\lambda\left(h_{i}\right)}-q_{i}^{-\lambda\left(h_{i}\right)}\right)-\left(q_{i}^{\lambda\left(h_{i}\right)-2}-q_{i}^{-\lambda\left(h_{i}\right)+2}\right)\right. \\
& \left.\quad+\cdots-\left(q_{i}^{-\lambda\left(h_{i}\right)+2}-q_{i}^{\lambda\left(h_{i}\right)-2}\right)+\left(q_{i}^{-\lambda\left(h_{i}\right)}-q_{i}^{\lambda\left(h_{i}\right)}\right)\right) f_{i}^{\lambda\left(h_{i}\right)} \cdot v_{\lambda}=0 .
\end{aligned}
$$

Note that we used the fact that $\lambda\left(h_{i}\right) \in 2 \mathbf{Z}_{\geq 0}$ in a critical way.

Finally, for $j \neq i$, we have $e_{j, \ell} f_{i, k}^{\lambda\left(h_{i}\right)+1} \cdot v_{\lambda}=\theta_{i, j}^{-\left(\lambda\left(h_{i}\right)+1\right)} f_{i, k}^{\lambda\left(h_{i}\right)+1} e_{j, \ell} \cdot v_{\lambda}=0$. Therefore, $f_{i, k}^{\lambda\left(h_{i}\right)+1} \cdot v_{\lambda}$ is a primitive vector of weight $\lambda-\left(\lambda\left(h_{i}\right)+1\right) \alpha_{i} \neq \lambda$, and hence $f_{i, k}^{\lambda\left(h_{i}\right)+1} \cdot v_{\lambda}=0$.

3.20. Assume $\lambda \in P$ and let $V^{q}$ be a highest weight module over $U_{q}(\mathfrak{g})$ with highest weight $\lambda$ and highest weight vector $v_{\lambda}$. We define the $\mathbf{A}$-form $V_{\mathbf{A}}^{q}$ of $V^{q}$ to be the $U_{\mathbf{A}}$-submodule of $V^{q}$ generated by $v_{\lambda}$; that is, $V_{\mathbf{A}}^{q}=U_{\mathbf{A}} \cdot v_{\lambda}$. 
Proposition 3.21. $V_{A}^{q}=U_{A}^{-} \cdot v_{\lambda}$.

Proof. Recall that every element $u$ of $U_{\mathbf{A}}$ can be written as a sum of elements of the form $u^{-} u^{0} u^{+}$, where $u^{0} \in U_{\mathbf{A}}^{0}, u^{ \pm} \in U_{\mathbf{A}}^{ \pm}$. By definition, $u^{+} \cdot v_{\lambda}=0$ unless $u^{+} \in \mathbf{A}$, and $q^{h} \cdot v_{\lambda}=q^{\lambda(h)} v_{\lambda} \in \mathbf{A} v_{\lambda}$. For $i \in I, c \in \mathbf{Z}$, and $n \in \mathbf{Z}_{>0}$, we have

$$
\left[\begin{array}{c}
K_{i} ; c \\
n
\end{array}\right] \cdot v_{\lambda}=\left[\begin{array}{c}
\lambda\left(h_{i}\right)+c \\
n
\end{array}\right]_{q_{i}} v_{\lambda}
$$

where

$$
\left[\begin{array}{c}
\lambda\left(h_{i}\right)+c \\
n
\end{array}\right]_{q_{i}}=\prod_{r=1}^{n} \frac{q_{i}^{\lambda\left(h_{i}\right)+c-r+1}-q_{i}^{-\left(\lambda\left(h_{i}\right)+c-r+1\right)}}{q_{i}^{r}-q_{i}^{-r}} .
$$

Since $\left[\begin{array}{c}\lambda\left(h_{i}\right)+c \\ n\end{array}\right]_{q_{i}} \in \mathbf{A}$, it follows that $\left[\begin{array}{c}K_{i} ; c \\ n\end{array}\right] \cdot v_{\lambda} \in \mathbf{A} v_{\lambda}$; similarly, $\left[\begin{array}{c}D_{i} ; c \\ n\end{array}\right] \cdot v_{\lambda}$ $\in \mathbf{A} v_{\lambda}$. Therefore $u^{-} u^{0} u^{+} \cdot v_{\lambda} \in \mathbf{A} u^{-} \cdot v_{\lambda} \subset U_{\mathbf{A}}^{-} \cdot v_{\lambda}$. Consequently, $V_{\mathbf{A}}^{q}=U_{\mathbf{A}}^{-} \cdot v_{\lambda}$ as claimed.

Proposition 3.22. The map $\phi: C(q) \otimes_{\boldsymbol{A}} V_{\boldsymbol{A}}^{q} \rightarrow V^{q}$ given by $f \otimes v \mapsto f v(f \in$ $\left.\boldsymbol{C}(q), v \in V_{A}^{q}\right)$ is an $\boldsymbol{C}(q)$-linear isomorphism.

Proof. It is clear that the $\mathbf{C}(q)$-linear map $\phi$ given above is surjective. Let $\left\{f_{\zeta} \cdot v_{\lambda} \mid \zeta \in \Omega\right\}$ be a basis of $V^{q}$, where $f_{\zeta}$ is a monomial in $f_{i, k}$ 's. Define a $\mathbf{C}(q)$-linear map $\psi: V^{q} \rightarrow \mathbf{C}(q) \otimes_{\mathbf{A}} V_{\mathbf{A}}^{q}$ by

$$
\psi\left(f_{\zeta} \cdot v_{\lambda}\right)=1 \otimes f_{\zeta} \cdot v_{\lambda} .
$$

Then it is easy to see that $\phi$ and $\psi$ are inverses of each other, which proves our assertion.

Proposition 3.23. For $\mu \in P$, let $\left(V_{A}^{q}\right)_{\mu}=V_{A}^{q} \cap V_{\mu}^{q}$. Then $V_{A}^{q}$ has the weight space decomposition, $V_{A}^{q}=\bigoplus_{\mu \in P}\left(V_{A}^{q}\right)_{\mu}$.

Proof. Assume $v=v_{1}+\cdots+v_{p} \in V_{\mathbf{A}}^{q}$, where $v_{j} \in V_{\mu_{j}}^{q}\left(\mu_{j} \in P, j=1, \cdots, p\right)$. We would like to prove that $v_{j} \in V_{\mathbf{A}}^{q}$ for all $j=1, \cdots, p$. We will show that $v_{1} \in V_{\mathbf{A}}^{q}$. The other cases can be verified in a similar way.

For $j=1,2, \cdots, p$ and $i \in I$, write $\mu_{j}\left(h_{i}\right)=S_{i, j}$ and $\mu_{j}\left(d_{i}\right)=T_{i, j}$. Since $\mu_{j} \neq \mu_{1}$ for $j=2, \cdots, p$, we can choose an index $i_{j} \in I$ such that $S_{i_{j}, j} \neq S_{i_{j}, 1}$ or $T_{i_{j}, j} \neq T_{i_{j}, 1}$. Let $I_{0}=\left\{i_{2}, i_{3}, \cdots, i_{p}\right\}$, and let $s$ be a positive integer such that $s \geq\left|S_{i, j}-S_{i, 1}\right|$ and $s \geq\left|T_{i, j}-T_{i, 1}\right|$ for all $i \in I_{0}, j=1, \cdots, p$. (Simply take the maximum of the numbers $\left|S_{i, j}-S_{i, 1}\right|,\left|T_{i, j}-T_{i, 1}\right|$ for $i \in I_{0}, j=1, \cdots, p$.) Let $u \in U_{\mathbf{A}}$ be defined by

$$
\begin{aligned}
& u=\prod_{i \in I_{0}}\left[\begin{array}{c}
K_{i} ;-S_{i, 1}+s \\
s
\end{array}\right]\left[\begin{array}{c}
K_{i} ;-S_{i, 1}-1 \\
s
\end{array}\right] \\
& \times\left[\begin{array}{c}
D_{i} ;-T_{i, 1}+s \\
s
\end{array}\right]\left[\begin{array}{c}
D_{i} ;-T_{i, 1}-1 \\
s
\end{array}\right] \text {. }
\end{aligned}
$$

Then we have

$$
\begin{aligned}
{\left[\begin{array}{c}
D_{i} ;-T_{i, 1}-1 \\
s
\end{array}\right] \cdot v_{1} } & =\prod_{r=1}^{s} \frac{D_{i} q_{i}^{-T_{i, 1}-r}-D_{i}^{-1} q_{i}^{T_{i, 1}+r}}{q_{i}^{r}-q_{i}^{-r}} v_{1} \\
& =\prod_{r=1}^{s} \frac{q_{i}^{-r}-q_{i}^{r}}{q_{i}^{r}-q_{i}^{-r}} v_{1}=(-1)^{s} v_{1}
\end{aligned}
$$


and

$$
\begin{aligned}
{\left[\begin{array}{c}
D_{i} ;-T_{i, 1}+s \\
s
\end{array}\right] \cdot v_{1} } & =\prod_{r=1}^{s} \frac{D_{i} q_{i}^{-T_{i, 1}+s-r+1}-D_{i}^{-1} q_{i}^{T_{i, 1}-s+r-1}}{q_{i}^{r}-q_{i}^{-r}} v_{1} \\
& =\prod_{r=1}^{s} \frac{q_{i}^{s-r+1}-q_{i}^{-s+r-1}}{q_{i}^{r}-q_{i}^{-r}} v_{1}=v_{1}
\end{aligned}
$$

Similarly,

$$
\left[\begin{array}{c}
K_{i} ;-S_{i, 1}-1 \\
s
\end{array}\right] \cdot v_{1}=(-1)^{s} v_{1}, \text { and }\left[\begin{array}{c}
K_{i} ;-S_{i, 1}+s \\
s
\end{array}\right] \cdot v_{1}=v_{1} .
$$

Therefore, $u \cdot v_{1}=(-1)^{2 s(p-1)} v_{1}=v_{1}$.

If $j \neq 1$, then

$$
\left[\begin{array}{c}
D_{i} ;-T_{i, 1}-1 \\
s
\end{array}\right] \cdot v_{j}=\prod_{r=1}^{s} \frac{q_{i}^{T_{i, j}-T_{i, 1}-r}-q_{i}^{-\left(T_{i, j}-T_{i, 1}-r\right)}}{q_{i}^{r}-q_{i}^{-r}} v_{j}
$$

and

$$
\left[\begin{array}{c}
D_{i} ;-T_{i, 1}+s \\
s
\end{array}\right] \cdot v_{j}=\prod_{r=1}^{s} \frac{q_{i}^{T_{i, j}-T_{i, 1}+s-r+1}-q_{i}^{-\left(T_{i, j}-T_{i, 1}+s-r+1\right)}}{q_{i}^{r}-q_{i}^{-r}} v_{j}
$$

Thus,

$$
\begin{aligned}
& \prod_{i \in I_{0}}\left[\begin{array}{c}
D_{i} ;-T_{i, 1}+s \\
s
\end{array}\right]\left[\begin{array}{c}
D_{i} ;-T_{i, 1}-1 \\
s
\end{array}\right] \cdot v_{j} \\
& =\prod_{i \in I_{0}} \prod_{r, t=1}^{s} \frac{\left(q_{i}^{T_{i, j}-T_{i, 1}-r}-q_{i}^{-\left(T_{i, j}-T_{i, 1}-r\right)}\right)\left(q_{i}^{T_{i, j}-T_{i, 1}+s-t+1}-q_{i}^{-\left(T_{i, j}-T_{i, 1}+s-t+1\right)}\right)}{\left(q_{i}^{r}-q_{i}^{-r}\right)\left(q_{i}^{t}-q_{i}^{-t}\right)} v_{j}
\end{aligned}
$$

Similarly,

$$
\begin{aligned}
& \prod_{i \in I_{0}}\left[\begin{array}{c}
K_{i} ; \\
-S_{i, 1}+s \\
s
\end{array}\right]\left[\begin{array}{c}
K_{i} ;-S_{i, 1}-1 \\
s
\end{array}\right] \cdot v_{j} \\
& =\prod_{i \in I_{0}} \prod_{r, t=1}^{s} \frac{\left(q_{i}^{S_{i, j}-S_{i, 1}-r}-q_{i}^{-\left(S_{i, j}-S_{i 1}-r\right)}\right)\left(q_{i}^{S_{i, j}-S_{i, 1}+s-t+1}-q_{i}^{-\left(S_{i, j}-S_{i, 1}+s-t+1\right)}\right)}{\left(q_{i}^{r}-q_{i}^{-r}\right)\left(q_{i}^{t}-q_{i}^{-t}\right)} v_{j}
\end{aligned}
$$

The terms where $r+t=s+1$ are

$$
\begin{aligned}
& \left(q_{i}^{T_{i, j}-T_{i, 1}-r}-q_{i}^{-\left(T_{i, j}-T_{i, 1}-r\right)}\right)\left(q_{i}^{T_{i, j}-T_{i, 1}+s-t+1}-q_{i}^{-\left(T_{i, j}-T_{i, 1}+s-t+1\right)}\right) \\
& \quad=q_{i}^{2\left(T_{i, j}-T_{i, 1}\right)}-q_{i}^{2 r}-q_{i}^{-2 r}+q_{i}^{-2\left(T_{i, j}-T_{i, 1}\right)}, \\
& \left(q_{i}^{S_{i, j}-S_{i, 1}-r}-q_{i}^{-\left(S_{i, j}-S_{i, 1}-r\right)}\right)\left(q_{i}^{S_{i, j}-S_{i, 1}+s-t+1}-q_{i}^{-\left(S_{i, j}-S_{i, 1}+s-t+1\right)}\right) \\
& \quad=q_{i}^{2\left(S_{i, j}-S_{i, 1}\right)}-q_{i}^{2 r}-q_{i}^{-2 r}+q_{i}^{-2\left(S_{i, j}-S_{i, 1}\right)} .
\end{aligned}
$$

By the definition of $I_{0}$, either $S_{i, j}-S_{i, 1} \neq 0$ or $T_{i, j}-T_{i, 1} \neq 0$ for $i=i_{j} \in I_{0}$. Thus, as $r$ runs from 1 to $s$, there is some value of $r$ such that $r=\left|S_{i, j}-S_{i, 1}\right|$ or $r=\left|T_{i, j}-T_{i, 1}\right|$ for $i=i_{j} \in I_{0}$, which implies $u \cdot v_{j}=0$. Therefore, $u \cdot v=v_{1}$, and hence $v_{1} \in V_{\mathbf{A}}^{q}$. 
Corollary 3.25. For all $\mu \in P,\left(V_{A}^{q}\right)_{\mu}$ is a free $\mathbf{A}$-module, and $\operatorname{rank}_{\boldsymbol{A}}\left(V_{A}^{q}\right)_{\mu}=$ $\operatorname{dim}_{C(q)} V_{\mu}^{q}$.

Proof. By Propositions 3.22 and 3.23, there is a $\mathbf{C}(q)$-linear isomorphism $\mathbf{C}(q) \otimes_{\mathbf{A}}$ $\left(V_{\mathbf{A}}^{q}\right)_{\mu} \cong V_{\mu}^{q}$ for all $\mu \in P$, from which the assertion follows.

\section{Classical Limits}

4.1. Recall that $\mathbf{A}=\mathbf{C}\left[q, q^{-1}, 1 /[n]_{q_{i}}, i \in I, n>0\right]$ and $U_{\mathbf{A}}$ is the $\mathbf{A}$-form in the quantum algebra $U_{q}(\mathfrak{g})$, where $\mathfrak{g}$ is the Borcherds superalgebra $\mathfrak{g}=\mathfrak{g}(A, \underline{m}, C)$. Let $\mathbf{J}$ be the ideal of $\mathbf{A}$ generated by $q-1$. Then there is an isomorphism of fields $\mathbf{A} / \mathbf{J} \cong \mathbf{C}$ given by $f+\mathbf{J} \mapsto f(1)$ for $f \in \mathbf{A}$. As before, suppose $V^{q}=U_{q}(\mathfrak{g}) v_{\lambda}$ is any highest weight module and $V_{\mathbf{A}}^{q}$ is the $U_{\mathbf{A}}$-submodule generated by $v_{\lambda}$ inside $V^{q}$. Set

$$
\begin{aligned}
& U \stackrel{\text { def }}{=}(\mathbf{A} / \mathbf{J}) \otimes_{\mathbf{A}} U_{\mathbf{A}}, \\
& V \stackrel{\text { def }}{=}(\mathbf{A} / \mathbf{J}) \otimes_{\mathbf{A}} V_{\mathbf{A}}^{q},
\end{aligned}
$$

and note that $U \cong U_{\mathbf{A}} / \mathbf{J} U_{\mathbf{A}}$ and $V \cong V_{\mathbf{A}}^{q} / \mathbf{J} V_{\mathbf{A}}^{q}$. For each $\mu \in P$, let $V_{\mu}=$ $\mathbf{A} / \mathbf{J} \otimes_{\mathbf{A}}\left(V_{\mathbf{A}}^{q}\right)_{\mu}$. Since $V_{\mathbf{A}}^{q}=\bigoplus_{\mu \in P}\left(V_{\mathbf{A}}^{q}\right)_{\mu}$, then $V=\bigoplus_{\mu \in P} V_{\mu}$, and the following holds:

Proposition 4.3. $\operatorname{dim}_{A / J} V_{\mu}=\operatorname{rank}_{A}\left(V_{A}^{q}\right)_{\mu}$.

Proof. If $\left\{v_{j} \mid j=1, \cdots, r\right\} \quad\left(r=\operatorname{rank}_{\mathbf{A}}\left(V_{\mathbf{A}}^{q}\right)_{\mu}\right)$ is a basis of the free A-module $\left(V_{\mathbf{A}}^{q}\right)_{\mu}$, then by $\left[\mathrm{H}\right.$, Theorem 5.11, Ch. 4] every element $v$ of $V_{\mu}=\mathbf{A} / \mathbf{J} \otimes_{\mathbf{A}}\left(V_{\mathbf{A}}^{q}\right)_{\mu}$ can be written uniquely as $v=\sum_{j=1}^{r} a_{j} \otimes v_{j}\left(a_{j} \in \mathbf{A} / \mathbf{J}\right)$. It follows that $\left\{\bar{v}_{j}=\right.$ $\left.1 \otimes v_{j} \mid j=1, \cdots, r\right\}$ is a basis of the $\mathbf{A} / \mathbf{J}$-vector space $V_{\mu}$.

4.4. Consider the natural maps $U_{\mathbf{A}} \longrightarrow U_{\mathbf{A}} / \mathbf{J} U_{\mathbf{A}} \cong U$ and $V_{\mathbf{A}}^{q} \longrightarrow V_{\mathbf{A}}^{q} / \mathbf{J} V_{\mathbf{A}}^{q} \cong V$. We see that $q \rightarrow 1$ under these mappings. We write $\bar{u}$ and $\bar{v}$ for the images of the elements $u \in U_{\mathbf{A}}$ and $v \in V_{\mathbf{A}}^{q}$, respectively. We also denote by $\overline{h_{i}}$ and $\overline{d_{i}}(i \in I)$ the images of $\left[\begin{array}{c}K_{i} ; 0 \\ 1\end{array}\right]=\frac{K_{i}-K_{i}^{-1}}{q_{i}-q_{i}^{-1}}$ and $\left[\begin{array}{c}D_{i} ; 0 \\ 1\end{array}\right]=\frac{D_{i}-D_{i}^{-1}}{q_{i}-q_{i}^{-1}}$, respectively. It is natural to expect that the algebra $U$ is isomorphic to the universal enveloping algebra $U(\mathfrak{g})$ of the Borcherds superalgebra $\mathfrak{g}$. We argue in the next lemma that ${\overline{K_{i}}}^{2}={\overline{D_{i}}}^{2}=1$ in $U$, but to obtain the desired isomorphism we have to factor out more from $U$.

Lemma 4.5. In the algebra $U$ we have ${\overline{K_{i}}}^{2}={\overline{D_{i}}}^{2}=1$ for all $i \in I$.

Proof. In $U_{\mathbf{A}}$ we have

$$
K_{i}-K_{i}^{-1}=\left(q_{i}-q_{i}^{-1}\right)\left[\begin{array}{c}
K_{i} ; 0 \\
1
\end{array}\right]=\left(q_{i}-1\right)\left(1+q_{i}^{-1}\right)\left[\begin{array}{c}
K_{i} ; 0 \\
1
\end{array}\right] .
$$

Thus, $\overline{K_{i}}-{\overline{K_{i}}}^{-1}=0 \cdot \overline{h_{i}}=0$ in $U$, which implies that ${\overline{K_{i}}}^{2}=1$. Similarly, we have ${\overline{D_{i}}}^{2}=1$ in $U$. 
4.6. Let $R$ be the ideal of $U$ generated by the elements $\overline{q^{h}}-1\left(h \in P^{\vee}\right)$, and set $U_{1}=U / R$. By abuse of notation, we will also use $\bar{u}$ for the image of the element $u \in U_{\mathbf{A}}$ in $U_{1}$, and $\overline{h_{i}}$ and $\overline{d_{i}}$ for the images of $\left[\begin{array}{c}K_{i} ; 0 \\ 1\end{array}\right]=\frac{K_{i}-K_{i}^{-1}}{q_{i}-q_{i}^{-1}}$ and $\left[\begin{array}{cc}D_{i} ; & 0 \\ 1\end{array}\right]=\frac{D_{i}-D_{i}^{-1}}{q_{i}-q_{i}^{-1}}$ in $U_{1}$, respectively. Then, since $\overline{q^{h}}=1$ in $U_{1}$, the algebra $U_{1}$ is generated by the elements $\overline{e_{i, k}}, \overline{f_{i, k}}, \overline{h_{i}}, \overline{d_{i}}\left(i \in I, k=1, \ldots, m_{i}\right)$. Note that $\overline{q^{h}} \in U$ acts as the identity on $V=\mathbf{A} / \mathbf{J} \otimes_{\mathbf{A}} V_{\mathbf{A}}^{q}$ for any highest weight module $V^{q}$ over $U_{q}(\mathfrak{g})$. Indeed, if $\left\{v_{j}\right\}_{j=1}^{r}\left(r=\operatorname{rank}_{\mathbf{A}}\left(V_{\mathbf{A}}^{q}\right)_{\mu}\right)$ is an $\mathbf{A}$-basis of $\left(V_{\mathbf{A}}^{q}\right)_{\mu}(\mu \in P)$, then by Proposition 4.3, $\left\{\overline{v_{j}}=1 \otimes v_{j}\right\}_{j=1}^{r}$ is an $\mathbf{A} / \mathbf{J}$-basis of $V_{\mu}$. Since $q^{h} \cdot v_{j}=q^{\mu(h)} v_{j}$ for all $h \in P^{\vee}, j=1, \ldots, r$, letting $q \rightarrow 1$, we obtain $\overline{q^{h}} \cdot \overline{v_{j}}=\overline{v_{j}}$ for all $j=1, \ldots, r$. Therefore, $\overline{q^{h}}=\operatorname{id}_{V_{\mu}}$ on $V_{\mu}(\mu \in P)$, and hence $\overline{q^{h}}$ is the identity transformation on $V$. It follows that $U_{1} \cdot \overline{v_{\lambda}}=U \cdot \overline{v_{\lambda}}=V$.

Consider the natural maps

$$
U_{\mathbf{A}} \rightarrow U_{\mathbf{A}} / \mathbf{J} U_{\mathbf{A}} \cong U \rightarrow U_{1}=U / R
$$

and

$$
V_{\mathbf{A}}^{q} \rightarrow V_{\mathbf{A}}^{q} / \mathbf{J} V_{\mathbf{A}}^{q} \cong V=U_{1} \cdot \overline{v_{\lambda}}
$$

The passage from $U_{\mathbf{A}}$ (resp. $V_{\mathbf{A}}^{q}$ ) to $U_{1}$ (resp. $V$ ) under these maps is referred to as taking the classical limit.

Theorem 4.7. (a) The elements $\overline{e_{i, k}}, \overline{f_{i, k}}, \overline{h_{i}}, \overline{d_{i}}\left(i \in I, k=1, \cdots, m_{i}\right)$ in $U_{1}$ satisfy the relations in (1.14). Hence as endomorphisms on $V$ they satisfy the relations in (1.14), and thus $V$ has a $U(\mathfrak{g})$-module structure.

(b) As a $U(\mathfrak{g})$-module, $V$ is a highest weight module with highest weight $\lambda \in P$ and highest weight vector $\overline{v_{\lambda}}=1 \otimes v_{\lambda}$.

(c) The endomorphisms $\overline{h_{i}}$ and $\overline{d_{i}}(i \in I)$ act on $V_{\mu}(\mu \in P)$ as scalar multiplication by $\mu\left(h_{i}\right)$ and $\mu\left(d_{i}\right)$, respectively. Therefore $V_{\mu}$ is the $\mu$-weight space of the $U(\mathfrak{g})$-module $V$.

Proof. (a) Since $\left[\frac{K_{i}-K_{i}^{-1}}{q_{i}-q_{i}^{-1}}, \frac{K_{j}-K_{j}^{-1}}{q_{j}-q_{j}^{-1}}\right]=0$ for $i, j \in I$, we obtain $\left[\overline{h_{i}}, \overline{h_{j}}\right]=0$ by letting $q \rightarrow 1$. Similarly, $\left[\overline{h_{i}}, \overline{d_{j}}\right]=0$ and $\left[\overline{d_{i}}, \overline{d_{j}}\right]=0$.

For $i, j \in I, k=1, \cdots, m_{j}$, we have

$$
\begin{aligned}
\frac{K_{i}-K_{i}^{-1}}{q_{i}-q_{i}^{-1}} & e_{j, k}-e_{j, k} \frac{K_{i}-K_{i}^{-1}}{q_{i}-q_{i}^{-1}} \\
= & \frac{K_{i}-K_{i}^{-1}}{q_{i}-q_{i}^{-1}} e_{j, k}-\frac{K_{i} q_{i}^{-a_{i, j}}-K_{i}^{-1} q^{a_{i, j}}}{q_{i}-q_{i}^{-1}} e_{j, k} \\
= & \frac{K_{i}\left(1-q_{i}^{-a_{i, j}}\right)-K_{i}^{-1}\left(1-q_{i}^{a_{i, j}}\right)}{q_{i}-q_{i}^{-1}} e_{j, k} \\
= & {\left[K_{i} \frac{q_{i}^{a_{i, j}}-q_{i}^{-a_{i, j}}}{q_{i}-q_{i}^{-1}}+\left(1-q_{i}^{a_{i, j}}\right) \frac{K_{i}-K_{i}^{-1}}{q_{i}-q_{i}^{-1}}\right] e_{j, k} . }
\end{aligned}
$$

Thus, taking the classical limit gives $\overline{h_{i}} \overline{e_{j, k}}-\overline{e_{j, k}} \overline{h_{i}}=a_{i, j} \overline{e_{j, k}}$ for $i, j \in I, k=$ $1, \cdots, m_{j}$. 
Similarly, we obtain

$$
\begin{aligned}
& \overline{h_{i} f_{j, k}}-\overline{f_{j, k} h_{i}}=-a_{i, j} \overline{f_{j, k}}, \\
& \overline{d_{i}} \overline{e_{j, k}}-\overline{e_{j, k}} \overline{d_{i}}=\delta_{i, j} \overline{e_{j, k}}, \\
& \overline{d_{i} f_{j, k}}-\overline{f_{j, k} d_{i}}=-\delta_{i, j} \overline{f_{j, k}} .
\end{aligned}
$$

The rest of the relations can be derived using the fact that

$$
\{n\}_{q_{i}} \longrightarrow\left\{\begin{array}{l}
n \text { if } i \text { is even, } \\
0 \text { if } i \text { is odd, } n \text { is even, } \\
1 \text { if } i \text { is odd, } n \text { is odd }
\end{array}\right.
$$

as $q \rightarrow 1\left(n \in \mathbf{Z}_{\geq 0}\right)$. Therefore, there exists a surjective algebra homomorphism $\psi: U(\mathfrak{g}) \longrightarrow U_{1}$ defined by $e_{i, k} \mapsto \overline{e_{i, k}}, f_{i, k} \mapsto \overline{f_{i, k}}, h_{i} \mapsto \overline{h_{i}}, d_{i} \mapsto \overline{d_{i}}(i \in I, k=$ $\left.1,2, \cdots, m_{i}\right)$, which can be used to give $V$ a $U(\mathfrak{g})$-module structure.

(b) It is clear that $\overline{e_{i, k}} \cdot \overline{v_{\lambda}}=0$ for $i \in I, k=1, \cdots, m_{i}$. Since

$$
\frac{K_{i}-K_{i}^{-1}}{q_{i}-q_{i}^{-1}} v_{\lambda}=\frac{q_{i}^{\lambda\left(h_{i}\right)}-q_{i}^{-\lambda\left(h_{i}\right)}}{q_{i}-q_{i}^{-1}} v_{\lambda},
$$

by taking the classical limit, we obtain $\overline{h_{i}} \cdot \overline{v_{\lambda}}=\lambda\left(h_{i}\right) \overline{v_{\lambda}}$ for $i \in I$. Similarly, we have $\overline{d_{i}} \cdot \overline{v_{\lambda}}=\lambda\left(d_{i}\right) \overline{v_{\lambda}}$ for $i \in I$. Recall that $V_{\mathbf{A}}^{q}=U_{\mathbf{A}}^{-} \cdot v_{\lambda}$, where $U_{\mathbf{A}}^{-}$is the A-subalgebra of $U_{\mathbf{A}}$ generated by the elements $f_{i, k}$ for $i \in I, k=1, \cdots, m_{i}$. Hence $V=U_{1}^{-} \cdot \overline{v_{\lambda}}$, where $U_{1}^{-}$is the $\mathbf{A} / \mathbf{J}$-subalgebra of $U_{1}$ with 1 generated by the elements $\overline{f_{i, k}}$ for $i \in I, k=1, \cdots, m_{i}$. Therefore, $V$ is a highest weight module over $U(\mathfrak{g})$ with highest weight $\lambda$ and highest weight vector $\overline{v_{\lambda}}$.

(c) For $v \in\left(V_{\mathbf{A}}^{q}\right)_{\mu}(\mu \in P)$ and $i \in I$, we have

$$
\frac{K_{i}-K_{i}^{-1}}{q_{i}-q_{i}^{-1}} v=\frac{q_{i}^{\mu\left(h_{i}\right)}-q_{i}^{-\mu\left(h_{i}\right)}}{q_{i}-q_{i}^{-1}} v,
$$

which yields $\overline{h_{i}} \cdot \bar{v}=\mu\left(h_{i}\right) \bar{v}$ by taking the classical limit. Similarly, $\overline{d_{i}} \cdot \bar{v}=\mu\left(d_{i}\right) \bar{v}$ $(i \in I)$.

4.8. We now prove our main results. We will show first that the irreducible highest weight module $V^{q}(\lambda)$ over $U_{q}(\mathfrak{g})$ with highest weight $\lambda \in P^{+}$is a quantum deformation of the irreducible highest weight module $V(\lambda)$ over $U(\mathfrak{g})$, and that they have the same character formula. Using this result, we will argue that the classical limit $U_{1}$ of the quantum group $U_{q}(\mathfrak{g})$ is isomorphic to the universal enveloping algebra $U(\mathfrak{g})$ of the Borcherds superalgebra $\mathfrak{g}$, and then prove that the Verma module $M(\lambda)$ over $U(\mathfrak{g})$ for $\lambda \in P$ also can be deformed to the Verma module $M_{q}(\lambda)$ over the quantum algebra $U_{q}(\mathfrak{g})$.

Theorem 4.9. If $\lambda \in P^{+}$and $V^{q}$ is the irreducible highest weight module $V^{q}(\lambda)$ over $U_{q}(\mathfrak{g})$ with highest weight $\lambda$, then $V$ is isomorphic to the irreducible highest weight module $V(\lambda)$ over $U(\mathfrak{g})$ with highest weight $\lambda$. Therefore, any irreducible highest weight module $V(\lambda)$ over $U(\mathfrak{g})$ with highest weight $\lambda \in P^{+}$admits a quantum deformation to the irreducible highest weight module $V^{q}(\lambda)$ over $U_{q}(\mathfrak{g})$ with highest weight $\lambda \in P^{+}$in such a way that the dimensions of weight spaces are invariant under the deformation. In particular, the character of $V^{q}(\lambda)$ is given by the WeylKac-Borcherds formula in Theorem 1.26. 
Proof. Let $v_{\lambda}$ be a highest weight vector of $V^{q}=V^{q}(\lambda)$. By Corollary 3.19, if $\lambda\left(h_{i}\right)=0$, then $f_{i, k} \cdot v_{\lambda}=0$ for $k=1, \cdots, m_{i}$, and if $a_{i, i}=2$, then $f_{i, k}^{\lambda\left(h_{i}\right)+1} \cdot v_{\lambda}=$ 0 . Letting $q \rightarrow 1$, we see that $V$ is a highest weight module over $U(\mathfrak{g})$ with highest weight $\lambda \in P^{+}$and highest weight vector $\overline{v_{\lambda}}$ that satisfies the conditions of Corollary 1.27. Therefore, $V$ is isomorphic to $V(\lambda)$. The second assertion follows from Corollary 3.25 and Proposition 4.3.

Corollary 4.10. Suppose that $\lambda \in P^{+}$and $V^{q}$ is a highest weight module over $U_{q}(\mathfrak{g})$ with highest weight $\lambda$ and highest weight vector $v_{\lambda}$ such that

(i) if $\lambda\left(h_{i}\right)=0$, then $f_{i, k} \cdot v_{\lambda}=0$ for $k=1, \cdots, m_{i}$,

(ii) if $a_{i, i}=2$, then $f_{i, k}^{\lambda\left(h_{i}\right)+1} \cdot v_{\lambda}=0$.

Then $V^{q}$ is isomorphic to the irreducible highest weight module $V^{q}(\lambda)$.

Proof. As we have seen in the proof of Theorem 4.7, if $V^{q}$ is a highest weight $U_{q}(\mathfrak{g})$ module satisfying the above conditions, then $V=\mathbf{A} / \mathbf{J} \otimes_{\mathbf{A}} V_{\mathbf{A}}^{q}$ is a highest weight $U(\mathfrak{g})$-module satisfying the conditions of Corollary 1.27 . Hence $V \cong V(\lambda)$, and $\operatorname{ch} V$ is given by the Weyl-Kac-Borcherds formula. By Corollary 3.25 and Proposition 4.3 , we have

$$
\operatorname{ch} V^{q}=\operatorname{ch} V=\operatorname{ch} V(\lambda)=\operatorname{ch} V^{q}(\lambda) .
$$

Therefore we have $V^{q} \cong V^{q}(\lambda)$.

Theorem 4.11. The classical limit $U_{1}$ of $U_{q}(\mathfrak{g})$ is isomorphic to the Hopf superalgebra $U(\mathfrak{g})$.

Proof. By Theorem 4.7 (a) the elements $\overline{e_{i, k}}, \overline{f_{i, k}}, \overline{h_{i}}, \overline{d_{i}}\left(i \in I, k=1, \cdots, m_{i}\right)$ in the algebra $U_{1}$ satisfy the relations in (1.14). Therefore, there exists a surjective algebra homomorphism $\psi: U(\mathfrak{g}) \longrightarrow U_{1}$ defined by $e_{i, k} \mapsto \overline{e_{i, k}}, f_{i, k} \mapsto \overline{f_{i, k}}, h_{i} \mapsto \overline{h_{i}}$, $d_{i} \mapsto \overline{d_{i}}\left(i \in I, k=1,2, \cdots, m_{i}\right)$.

Let $U_{1}^{0}$ be the subalgebra of $U_{1}$ generated by $\bar{h}_{i}, \bar{d}_{i}(i \in I)$, and $U_{1}^{+}$(resp. $U_{1}^{-}$) be the subalgebra of $U_{1}$ generated by $\bar{e}_{i, k}\left(\right.$ resp. $\left.\bar{f}_{i, k}\right)\left(i \in I, k=1,2, \cdots, m_{i}\right)$. We first show that the restriction $\psi_{0}$ of $\psi$ to $U(\mathfrak{h})$ is an isomorphism of $U(\mathfrak{h})$ onto $U_{1}^{0}$. Suppose $g \in \operatorname{Ker} \psi_{0}$, a polynomial in $h_{i}$ and $d_{i}(i \in I)$. For any linear functional $\lambda \in \mathfrak{h}^{*}$, we have $0=\bar{g} \cdot \bar{v}_{\lambda}=\lambda(g) \bar{v}_{\lambda}$, where $\bar{v}_{\lambda}$ is the highest weight vector of the $U_{1}$-module $V$ having highest weight $\lambda$, and $\lambda(g)$ denotes the polynomial in $\lambda\left(h_{i}\right)$ and $\lambda\left(d_{i}\right)$ corresponding to $g$. Hence, we have $\lambda(g)=0$ for all $\lambda \in \mathfrak{h}^{*}$. By assigning various values to the $\lambda\left(h_{i}\right)$ 's and $\lambda\left(d_{i}\right)$ 's, we can argue that $g=0$, which implies that $U_{1}^{0}$ is isomorphic to $U(\mathfrak{h})$.

Next, we would like to show that the restriction of $\psi$ to $U\left(\mathfrak{g}^{-}\right)$, which will be denoted by $\psi_{-}$, is an isomorphism of $U\left(\mathfrak{g}^{-}\right)$onto $U_{1}^{-}$. Suppose $\operatorname{Ker} \psi_{-} \neq 0$ and $u=\sum a_{\zeta} f_{\zeta} \in \operatorname{Ker} \psi_{-}$, where $a_{\zeta} \in \mathbf{C}$ and $f_{\zeta}$ are monomials in the $f_{i, k}$ 's. Let $N$ be the maximal length of the monomials $f_{\zeta}$ in the expression of $u$, and choose a dominant integral weight $\lambda \in P^{+}$such that $\lambda\left(h_{i}\right)>N$ for all $i \in I$. If $V^{q}=V^{q}(\lambda)$ is the irreducible $U_{q}(\mathfrak{g})$-module with highest weight $\lambda$, then by Theorem 4.9, the representation $\varphi: U(\mathfrak{g}) \longrightarrow V$ with $x \mapsto \psi(x) \cdot v_{\lambda}$ gives the irreducible $U(\mathfrak{g})$ module $V(\lambda)$ with highest weight $\lambda$. By Corollary 1.27 and our assumption on $\lambda$, the kernel of $\varphi$ restricted to $U\left(\mathfrak{g}^{-}\right)$is the left ideal of $U\left(\mathfrak{g}^{-}\right)$generated by the elements $f_{i, k}^{\lambda\left(h_{i}\right)+1}$ for $i \in I^{r e}$. Therefore, $u=\sum a_{\zeta} f_{\zeta}$ does not belong to $\operatorname{Ker} \varphi$. That is, $u \cdot v_{\lambda} \neq 0$. But, since $U\left(\mathfrak{g}^{-}\right)$acts on $V$ via the homomorphism $\psi_{-}$, we 
must have $u \cdot v_{\lambda}=\psi_{-}(u) \cdot v_{\lambda}=0$, which is a contradiction. Therefore, $\operatorname{Ker} \psi_{-}=0$, and hence $U\left(\mathfrak{g}^{-}\right)$is isomorphic to $U_{1}^{-}$. By the triangular decomposition we have

$$
U(\mathfrak{g}) \cong U\left(\mathfrak{g}^{-}\right) \otimes U(\mathfrak{h}) \otimes U\left(\mathfrak{g}^{+}\right) \cong U_{1}^{-} \otimes U_{1}^{0} \otimes U_{1}^{+} \cong U_{1} .
$$

Now it follows from Proposition 2.9 and the relations

$$
\begin{aligned}
& \Delta\left(\left[\begin{array}{c}
K_{i} ; 0 \\
1
\end{array}\right]\right)=\left[\begin{array}{c}
K_{i} ; 0 \\
1
\end{array}\right] \otimes K_{i}+K_{i}^{-1} \otimes\left[\begin{array}{c}
K_{i} ; 0 \\
1
\end{array}\right], \\
& \Delta\left(\left[\begin{array}{c}
D_{i} ; 0 \\
1
\end{array}\right]\right)=\left[\begin{array}{c}
D_{i} ; 0 \\
1
\end{array}\right] \otimes D_{i}+D_{i}^{-1} \otimes\left[\begin{array}{cc}
D_{i} ; & 0 \\
1
\end{array}\right], \\
& \varepsilon\left(\left[\begin{array}{c}
K_{i} ; 0 \\
1
\end{array}\right]\right)=0=\varepsilon\left(\left[\begin{array}{cc}
D_{i} ; & 0 \\
1
\end{array}\right]\right), \\
& S\left(\left[\begin{array}{c}
K_{i} ; 0 \\
1
\end{array}\right]\right)=-\left[\begin{array}{c}
K_{i} ; 0 \\
1
\end{array}\right], \quad S\left(\left[\begin{array}{c}
D_{i} ; 0 \\
1
\end{array}\right]\right)=-\left[\begin{array}{c}
D_{i} ; 0 \\
1
\end{array}\right]
\end{aligned}
$$

that $\Delta: U_{\mathbf{A}} \rightarrow U_{\mathbf{A}} \otimes U_{\mathbf{A}}, \varepsilon: U_{\mathbf{A}} \rightarrow \mathbf{A}$ and $S: U_{\mathbf{A}} \rightarrow U_{\mathbf{A}}$. Thus, by tensoring these mappings with the identity map on $\mathbf{A} / \mathbf{J}$, we obtain mappings on $U$, which we again denote $\Delta, \varepsilon$, and $S$, giving $U$ a Hopf superalgebra structure. In particular, in the algebra $U_{1}$ we have

$$
\begin{aligned}
& \Delta\left(\bar{h}_{i}\right)=\bar{h}_{i} \otimes 1+1 \otimes \bar{h}_{i}, \\
& \Delta\left(\bar{d}_{i}\right)=\bar{d}_{i} \otimes 1+1 \otimes \bar{d}_{i}, \\
& \Delta\left(\overline{e_{i, k}}\right)=\overline{e_{i, k}} \otimes 1+1 \otimes \overline{e_{i, k}}, \\
& \Delta\left(\overline{f_{i, k}}\right)=\overline{f_{i, k}} \otimes 1+1 \otimes \overline{f_{i, k}},
\end{aligned}
$$

and $\varepsilon(\bar{x})=0, S(\bar{x})=-\bar{x}$ for $\bar{x}=\overline{e_{i, k}}, \overline{f_{i, k}}, \bar{h}_{i}, \bar{d}_{i},\left(i \in I, k=1, \cdots, m_{i}\right)$. Therefore, the algebra $U_{1}$ has the Hopf superalgebra structure whose comultiplication, counit, and antipode are given by (4.13). The universal enveloping algebra $U(\mathfrak{g})$ of $\mathfrak{g}$ also has a Hopf superalgebra structure $\left(\Delta^{\bullet}, \epsilon^{\bullet}, S^{\bullet}\right)$. The generators $x=e_{i, k}, f_{i, k}, h_{i}$, $d_{i},\left(i \in I, k=1, \cdots, m_{i}\right)$ are primitive elements, (i.e. $\left.\Delta^{\bullet}(x)=x \otimes 1+1 \otimes x\right)$, and satisfy $\epsilon^{\bullet}(x)=0, S^{\bullet}(x)=-x$. Thus, we see that the homomorphism $\psi: U(\mathfrak{g}) \longrightarrow$ $U_{1}$ is a Hopf superalgebra isomorphism.

Remark 4.14. For generalized Kac-Moody (Borcherds) algebras $\mathfrak{g}$, it was asserted in $[\mathrm{Kn}]$ that the classical limit of the deformation $U_{q}(\mathfrak{g})$ is $U(\mathfrak{g})$, but no proof was given there. That result can be viewed as a special case of the one we just proved by taking $\theta_{i, j}=1$ for all $i, j \in I$.

Theorem 4.15. If $V^{q}$ is the Verma module $M^{q}(\lambda)$ over $U_{q}(\mathfrak{g})$ with highest weight $\lambda \in P$, then $V$ is isomorphic to the Verma module $M(\lambda)$ over $U(\mathfrak{g})$ with highest weight $\lambda$. Therefore, any Verma module $M(\lambda)$ over $U(\mathfrak{g})$ with highest weight $\lambda \in P$ admits a quantum deformation to the Verma module $M^{q}(\lambda)$ over $U_{q}(\mathfrak{g})$ with highest weight $\lambda \in P$ in such a way that the dimensions of weight spaces are invariant under the deformation.

Proof. Let $v_{\lambda}$ be a highest weight vector of $V^{q}$. It suffices to prove that $V$ is a free $U_{1}^{-}$-module of rank one generated by the vector $\overline{v_{\lambda}}$. Now $V^{q}=M^{q}(\lambda)$ is a free $U_{q}^{-}$-module of rank one generated by the highest weight vector $v_{\lambda}$. Thus by Propositions 3.21 and 3.22 , the $\mathbf{A}$-form $V_{\mathbf{A}}^{q}$ of $V^{q}$ is also a free $U_{\mathbf{A}}^{-}$-module generated by $v_{\lambda}$. Since $V_{\mathbf{A}}^{q}=U_{\mathbf{A}}^{-} \cdot v_{\lambda}$, by taking the classical limit we have $V=U_{1}^{-} \overline{v_{\lambda}}=U^{-} \overline{v_{\lambda}}$, where $U^{-}=\mathbf{A} / \mathbf{J} \otimes_{\mathbf{A}} U_{\mathbf{A}}^{-} \cong U_{\mathbf{A}}^{-} / \mathbf{J} U_{\mathbf{A}}^{-}$is the $\mathbf{A} / \mathbf{J}$-subalgebra of $U$ with 1 generated 
by $f_{i, k}$ for $i \in I, k=1, \cdots, m_{i}$. Suppose $\bar{u} \cdot \overline{v_{\lambda}}=0$ for some $\bar{u} \in U^{-}$. Then $\left(u+\mathbf{J} U_{\mathbf{A}}^{-}\right) \cdot\left(v_{\lambda}+\mathbf{J} V_{\mathbf{A}}^{q}\right)=u \cdot v_{\lambda}+\mathbf{J} V_{\mathbf{A}}^{q} \in \mathbf{J} V_{\mathbf{A}}^{q}=\mathbf{J} U_{\mathbf{A}}^{-} \cdot v_{\lambda}$, so that $u \cdot v_{\lambda}=u^{\prime} \cdot v_{\lambda}$ for some $u^{\prime} \in \mathbf{J} U_{\mathbf{A}}^{-}$. But since $V_{\mathbf{A}}^{q}$ is a free $U_{\mathbf{A}}^{-}$-module generated by $v_{\lambda}$, we must have $u=u^{\prime}$, which implies $\bar{u}=0$ in $U^{-}$. Therefore, $V$ is a free $U^{-}$-module generated by $\overline{v_{\lambda}}$. Since $U_{1}^{-} \cong U^{-}$and $V=U_{1}^{-} \cdot \overline{v_{\lambda}}=U^{-} \cdot \overline{v_{\lambda}}, V$ is a free $U_{1}^{-}$-module generated by $\overline{v_{\lambda}}$, and hence $V \cong M(\lambda)$. The second assertion is a consequence of Corollary 3.25 and Proposition 4.3.

\section{REFERENCES}

[BMPZ] Bahturin, Y.A., Mikhalev, A.A., Petrogradsky, V.M., Zaicev, M.V., Infinite Dimensional Lie Superalgebras, de Gruyter, Berlin, 1992. MR 94b:17001

[B1] Borcherds, R.E., Generalized Kac-Moody algebras, J. Algebra 115 (1988), 501-512. MR 89g:17004

[B2] Borcherds, R.E., The monster Lie algebra, Adv. Math. 83 (1990), 30-47. MR 90k:17027

[B3] Borcherds, R.E., Central extensions of generalized Kac-Moody algebras, J. Algebra 140 (1991), 330-335. MR 92g:17031

[B4] Borcherds, R.E., Monstrous moonshine and monstrous Lie superalgebras, Invent. Math. 109 (1992), 405-444. MR 94f: 11030

[B5] Borcherds, R.E., A characterization of generalized Kac-Moody algebras, J. Algebra 174 (1995), 1073-1079. MR 96e:17058

[CP] Chari, V., Pressley, A., A Guide to Quantum Groups, Cambridge University Press, Cambridge, 1994. MR 95j:17010; MR 96h:17014

[D] Drinfel'd, V.G., Hopf algebras and the quantum Yang-Baxter equation, Soviet Math. Dokl. 32 (1985), 254-258. MR 87h:58080

[FLV] Floreanini, R., Leites, D.A., Vinet, L., On the defining relations of quantum superalgebras, Lett. Math. Phys. 23 (1991), 127-131. MR 92m:17010

[G] Gebert, R.W., Introduction to vertex algebras, Borcherds algebras and the monster Lie algebra, Int. J. Mod. Phys. A 8 (1993), 5441-5503. MR 95a:17037

[H] Hungerford, T., Algebra, 5th ed., Springer-Verlag, New York, 1989. MR 82a:00006 (earlier ed.)

[Ja] Jantzen, J.C., Lectures on Quantum Groups, Amer. Math. Soc. Graduate Studies in Math., vol. 6, Providence, 1996. MR 96m:17029

[Ji] Jimbo, M., A q-difference analogue of $U(\mathfrak{g})$ and the Yang-Baxter equation, Lett. Math. Phys. 10 (1985), 63-69. MR 86k:17008

[Kc1] Kac, V.G., Lie superalgebras, Adv. Math. 26 (1977), 8-96. MR 58:5803

[Kc2] Kac, V.G., Infinite-dimensional algebras, Dedekind's $\eta$-function, classical Möbius function and the very strange formula, Adv. Math. 30 (1978), 85-136; 35 (1980), 264-273. MR 83a:17014a,b

[Kc3] Kac, V.G., Infinite Dimensional Lie Algebras, 3rd ed., Cambridge University Press, Cambridge, 1990. MR 92k:17038

[KW1] Kac, V.G., Wakimoto, M., Modular invariant representations of infinite-dimensional Lie algebras and superalgebras, Proc. Nat. Acad. Sci. U.S.A. 85 (1988), 4956-4960. MR 89j:17019

[KW2] Kac, V.G., Wakimoto, M., Integrable highest weight modules over affine superalgebras and number theory, Lie Theory and Geometry, Progress in Math., 123, Birkhäuser, Boston, 1994, pp. 415-456. MR 96j:11056

[Kn] Kang, S.-J., Quantum deformations of generalized Kac-Moody algebras and their modules, J. Algebra 175 (1995), 1041-1066. MR 96k:17023

[Ks] Kassel, C., Quantum Groups, Springer-Verlag, New York, 1995. MR 96e:17041

[KT] Khoroshkin, S.M., Tolstoy, V.N., Universal R-matrix for quantized (super)algebras, Comm. Math. Phys. 141 (1991), 599-617. MR 93a:16031

[L1] Lusztig, G., Quantum deformations of certain simple modules over enveloping algebras, Adv. Math. 70 (1988), 237-249. MR 89k:17029

[L2] Lusztig, G., Introduction to Quantum Groups, Birkhäuser, Boston, 1993. MR 94m:17016 
[M] Miyamoto, M., A generalization of Borcherds algebra and denominator formula, J. Algebra 180 (1996), 631-651. MR 97a:17026

[O] Olshanski, G.I., Quantized universal enveloping superalgebra of type $Q$ and a superextension of the Hecke algebra, Lett. in Math. Phys. 24 (1992), 93-102. MR 93i:17004

[Ra] Ray, U., A character formula for generalized Kac-Moody superalgebras, J. Algebra 177 (1995), 154-163. MR 97f:17008

[Ro] Rosso, M., Finite dimensional representations of the quantum analog of the enveloping algebra of a complex simple Lie algebra, Comm. Math. Phys. 117 (1988), 581-593. MR 90k:17019

[S1] Scheunert, M., Generalized Lie algebras, J. Math. Phys. A 20 (1979), 712-720. MR 80f: 17007

[S2] Scheunert, M., The presentation and $q$ deformation of special linear Lie superalgebras, J. Math. Phys. 34 (1993), 3780-3808. MR 94i:17007

[Y] Yamane, H., A Serre type theorem for affine Lie superalgebras and their quantized enveloping superalgebras, Proc. Japan Acad. Ser. A 70 (1994), 31-36. MR 95g:17021

[Z] Zou, Y.-M., Deformations of enveloping algebra of Lie superalgebra sl(m,n), Comm. Math. Phys. 162 (1994), 467-479. MR 95h:17014

Department of Mathematics, University of Wisconsin, Madison, Wisconsin 53706-1388

E-mail address: benkart@math.wisc.edu

Department of Mathematics, College of Natural Sciences, Seoul National UniverSity, SeOul 151-742, KoreA

E-mail address: sjkang@math.snu.ac.kr

Department of Mathematics, St. Lawrence University, Canton, New York 13617

E-mail address: dmel@music.stlawu.edu 